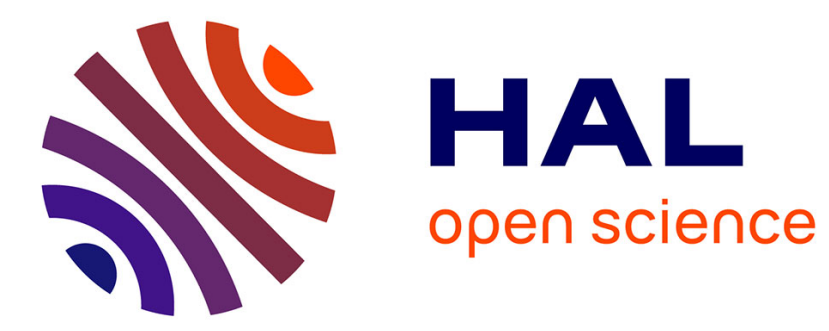

\title{
Stability of planar traveling fronts in bistable reaction-diffusion systems
}

\author{
Wei-Jie Sheng
}

\section{To cite this version:}

Wei-Jie Sheng. Stability of planar traveling fronts in bistable reaction-diffusion systems. Nonlinear Analysis: Hybrid Systems, 2017, 156, pp.42 - 60. 10.1016/j.na.2017.02.012 . hal-01583339

\section{HAL Id: hal-01583339 \\ https://hal.science/hal-01583339}

Submitted on 4 May 2021

HAL is a multi-disciplinary open access archive for the deposit and dissemination of scientific research documents, whether they are published or not. The documents may come from teaching and research institutions in France or abroad, or from public or private research centers.
L'archive ouverte pluridisciplinaire HAL, est destinée au dépôt et à la diffusion de documents scientifiques de niveau recherche, publiés ou non, émanant des établissements d'enseignement et de recherche français ou étrangers, des laboratoires publics ou privés. 


\title{
Stability of planar traveling fronts in bistable reaction-diffusion systems
}

\author{
Wei-Jie Sheng* \\ Department of Mathematics, Harbin Institute of Technology, Harbin, Heilongjiang 150001 \\ People's Republic of China \\ Aix Marseille Université, CNRS, Centrale Marseille, Institut de Mathématiques de Marseille, UMR 7373, \\ 13453 Marseille, France
}

\begin{abstract}
This paper is concerned with the multidimensional stability of planar traveling fronts in bistable reaction-diffusion systems. It is first shown that planar traveling fronts are asymptotically stable under spatially decaying initial perturbations by appealing to the comparison principle and super-subsolution method. In particular, if the perturbations belong to $L^{1}\left(\mathbb{R}^{n-1}\right)$ in a certain sense, we obtain a convergence rate like $t^{-\frac{n-1}{2}}$. Then we show that the solution of the Cauchy problem converges to the planar traveling front with rate $t^{-\frac{n+1}{4}}$ for a spatially non-decaying perturbation with the help of semigroup theory. Finally, we prove that there exists a solution oscillating permanently between two planar traveling fronts, which indicates that planar traveling fronts are not always asymptotically stable in multidimensional space under general bounded perturbations.
\end{abstract}

\section{Introduction}

In this paper, we study the large time behavior of the following Cauchy problem:

$$
\begin{cases}\frac{\partial \mathbf{u}}{\partial t}=\Delta \mathbf{u}+\mathbf{f}(\mathbf{u}), & \mathbf{x}=\left(x_{1}, \ldots, x_{n}\right), \quad t>0 \\ \mathbf{u}(\mathbf{x}, 0)=\mathbf{u}_{0}(\mathbf{x}), & \mathbf{x}=\left(x_{1}, \ldots, x_{n}\right),\end{cases}
$$

where $\mathbf{u}=\mathbf{u}(\mathbf{x}, t)=\left(u_{1}, \ldots, u_{m}\right) \in \mathbb{R}^{m},(\mathbf{x}, t) \in \mathbb{R}^{n} \times \mathbb{R}^{+}$with $n \geq 2$. In the sequel, we assume that $f$ satisfies the following hypotheses.

* Correspondence to: Department of Mathematics, Harbin Institute of Technology, Harbin, Heilongjiang 150001, People's Republic of China.

E-mail address: shengwj09@hit.edu.cn. 
(H1) There exist only two equilibrium $\mathbf{E}^{-}<\mathbf{E}^{+}$of $\mathbf{f}$, and $\mathbf{E}^{ \pm}$are stable. That is, $\mathbf{f}\left(\mathbf{E}^{ \pm}\right)=\mathbf{0}, \lambda_{ \pm}:=$ $s\left(\mathbf{f}^{\prime}\left(\mathbf{E}^{ \pm}\right)\right)<0$, where $s(\mathbf{A}):=\max \{\operatorname{Re} \lambda \mid \operatorname{det}(\lambda \mathbf{I}-\mathbf{A})=0\}$. We also assume that the matrices $\mathbf{f}^{\prime}\left(\mathbf{E}^{ \pm}\right)$ are irreducible.

(H2) The nonlinearity $\mathbf{f}(\mathbf{u})=\left(f_{1}(\mathbf{u}), \ldots, f_{m}(\mathbf{u})\right)$ is defined on an open domain $\Omega \subset \mathbb{R}^{n}$ and of class $C^{1+\alpha}$ in $\mathbf{u}$. Moreover, $\mathbf{f}$ satisfies the following conditions:

$$
\frac{\partial f_{i}}{\partial u_{j}} \geq 0 \quad \text { for all } \mathbf{u} \subset\left[\mathbf{E}^{-}, \mathbf{E}^{+}\right] \subset \Omega \text { and } 1 \leq i \neq j \leq m .
$$

Moreover, there exist nonnegative constants $L_{i j}^{ \pm}$such that

$$
\frac{\partial f_{i}}{\partial u_{j}}+L_{i j}^{-}\left\{u_{i}-E_{i}^{-}\right\}^{-}+L_{i j}^{+}\left\{E_{i}^{+}-u_{i}\right\}^{-} \geq 0 \quad \text { for } i \neq j \text { and } \mathbf{u} \in\left[\widehat{\mathbf{E}}^{-}, \widehat{\mathbf{E}}^{+}\right] \subset \Omega,
$$

where $\widehat{\mathbf{E}}^{-}<\mathbf{E}^{-}<\mathbf{E}^{+}<\widehat{\mathbf{E}}^{+}$and for any $a \in \mathbb{R}$,

$$
\{a\}^{-}= \begin{cases}0, & a \geq 0 \\ -a, & a<0\end{cases}
$$

According to [19], we define a function $\widetilde{\mathbf{f}}=\left(\widetilde{f}_{1}, \ldots, \widetilde{f}_{m}\right)$ as

$$
\widetilde{f}_{i}(\mathbf{u})=f_{i}(\mathbf{u})+\sum_{1 \leq j \leq m, j \neq i} L_{i j}^{-}\left\{u_{i}-E_{i}^{-}\right\}^{-}\left(u_{j}-E_{j}^{-}\right)+\sum_{1 \leq j \leq m, j \neq i} L_{i j}^{+}\left\{E_{i}^{+}-u_{i}\right\}^{+}\left(u_{j}-E_{j}^{+}\right)
$$

for $\mathbf{u} \subset\left[\widehat{\mathbf{E}}^{-}, \widehat{\mathbf{E}}^{+}\right]$. By Theorem 2.2 and Corollaries 1 and 2 in [19], the comparison principle follows immediately on $\left[\mathbf{E}^{-}, \mathbf{E}^{+}\right]$.

Here, we state some notations which will be used in this paper. For two vectors $\mathbf{c}=\left(c_{1}, \ldots, c_{m}\right)$ and $\mathbf{d}=\left(d_{1}, \ldots, d_{m}\right)$, the symbol $\mathbf{c}<\mathbf{d}$ means $c_{i}<d_{i}$ for each $i=1, \ldots, m$ and $\mathbf{c} \leq \mathbf{d}$ means $c_{i} \leq d_{i}$ for each $i=1, \ldots, m$. The interval $[\mathbf{c}, \mathbf{d}]$ denotes the set of $\mathbf{u} \in \mathbb{R}^{m}$ with $\mathbf{c} \leq \mathbf{u} \leq \mathbf{d}$. For $\mathbf{c}=\left(c_{1}, \ldots, c_{m}\right)$, we define $|\mathbf{c}|=\sum_{i=1}^{m} c_{i}^{2}$. For any bounded $\mathbf{u} \in C\left(\mathbb{R}^{n}, \mathbb{R}^{m}\right)$, we define $\|\mathbf{u}\|=\sup _{\mathbf{x} \in \mathbb{R}^{n}}|\mathbf{u}(\mathbf{x})|$. For simplicity, we use $\mathbf{0}$ and 1 to denote column vectors $(0, \ldots, 0)$ and $(1, \ldots, 1)$, respectively. In addition, $\Delta, \Delta_{\mathbf{x}^{\prime}}$ and $\nabla_{\mathbf{x}^{\prime}}$ refer to $\sum_{i=1}^{n-1} \frac{\partial^{2}}{\partial x_{i}^{2}}+\frac{\partial^{2}}{\partial \xi^{2}}, \sum_{i=1}^{n-1} \frac{\partial^{2}}{\partial x_{i}^{2}}$ and $\left(\frac{\partial}{\partial x_{1}}, \ldots, \frac{\partial}{\partial x_{n-1}}\right)$, respectively.

It is known from [18] that the one dimensional problem

$$
\frac{\partial \mathbf{u}}{\partial t}=\Delta \mathbf{u}+\mathbf{f}(\mathbf{u}), \quad(x, t) \in \mathbb{R} \times \mathbb{R}^{+}
$$

admits a planar traveling front

$$
\Phi(x+c t)=\left(\phi_{1}(x+c t), \ldots, \phi_{m}(x+c t)\right)
$$

satisfying

$$
\left\{\begin{array}{l}
\phi_{i}^{\prime \prime}-c \phi_{i}^{\prime}+f_{i}(\Phi)=0 \\
\Phi( \pm \infty):=\lim _{\xi \rightarrow \pm \infty} \Phi(\xi)=\mathbf{E}^{ \pm} \\
\phi_{i}^{\prime}>0
\end{array}\right.
$$

for $i=1, \ldots, m$, where $\xi=x+c t$. It is evident that such a front is also a solution of (1.1).

Stability is an important topic in the study of traveling fronts of reaction-diffusion equations [18]. Recently, an increasing attention has been paid to the study of multidimensional stability of traveling fronts. 
For instance, Xin [20] showed that the stability in one space dimension implies the stability in multiple dimensions for a scalar reaction-diffusion equation when $n \geq 4$ with perturbations decaying like $t^{-(n-1) / 4}$ by appealing to the theory of semigroups. Levermore and Xin [8] further studied the same problem with the help of the maximum principle and energy methods, they obtained that the planar traveling front is stable in any compact set moving with the wave for $n \geq 2$. Kapitula [6] extended the result in [20] to reaction-diffusion systems for the case $n \geq 2$ by considering a drift of perturbations along translates of the wave under a spectral hypothesis on the one-dimensional linearized operator around the traveling front (see Hypothesis 1.1 in [6]). Matano et al. [13] treated the $L^{\infty}$-stability in multidimensional space to Allen-Cahn equations under general initial perturbations. In particular, they proved that if the initial perturbations belong to $L^{1} \cap L^{\infty}$ in a certain sense, then the solution of the Cauchy problem goes to the planar traveling front algebraically. Zeng [21] considered the multidimensional stability of bistable reaction-diffusion equations and got an algebraic rate as time goes to infinity of planar traveling fronts in $L^{\infty}$. Lv and Wang [11] generalized the results in [13] to two species Lotka-Volterra competition-diffusion system. Other related works can be referred to $[1,16,17,15,12,10,11]$ and references therein.

Nevertheless, it seems that there is no research on the multidimensional stability for a general reaction-diffusion system without assuming the hypothesis on the spectral gap of the one-dimensional linearized operator in the unweighed space. The objective of the current study is to deal with the stability of planar traveling fronts in multiple dimensions. More precisely, we first prove that the planar traveling front is asymptotically stable under spatially decaying initial perturbations. In particular, we obtain an algebraic convergence rate $\left(t^{-\frac{n-1}{2}}\right)$ if the initial perturbations belong to $L^{1}$ in some sense. Then we further show that the solution of the Cauchy problem converges to the planar traveling front with rate $t^{-\frac{1}{2}}$ if $n=2,3$ and $t^{-\frac{n+1}{4}}$ if $n \geq 4$ for a spatially non-decaying perturbation with the help of semigroup theory. Finally, we prove that there exists a solution oscillating permanently between two planar traveling fronts, which indicates that planar traveling fronts are not always asymptotically stable in multidimensional space under general bounded initial perturbations.

Hereafter, we study system (1.1) in a reference frame. Without loss of generality, we assume that the solution travels towards $x_{n}$-direction. Let

$$
\mathbf{w}\left(\mathbf{x}^{\prime}, \xi, t\right)=\mathbf{u}\left(\mathbf{x}^{\prime}, x_{n}+c t, t\right), \quad \mathbf{x}^{\prime}=\left(x_{1}, \ldots, x_{n-1}\right), \xi=x_{n}+c t .
$$

Then $\mathbf{w}\left(\mathbf{x}^{\prime}, \xi, t\right)$ satisfies

$$
\begin{aligned}
& \mathbf{w}_{t}-\Delta \mathbf{w}+c \mathbf{w}_{\xi}-\mathbf{f}(\mathbf{w})=\mathbf{0}, \quad \mathbf{x}^{\prime} \in \mathbb{R}^{n-1}, \quad \xi \in \mathbb{R}, t>0, \\
& \mathbf{w}\left(\mathbf{x}^{\prime}, \xi, 0\right)=\mathbf{u}_{0}\left(\mathbf{x}^{\prime}, \xi\right), \quad \mathbf{x}^{\prime} \in \mathbb{R}^{n-1}, \xi \in \mathbb{R} .
\end{aligned}
$$

For simplicity, we still denote $\mathbf{w}\left(\mathbf{x}^{\prime}, \xi, t\right)$ by $\mathbf{u}\left(\mathbf{x}^{\prime}, \xi, t\right)$ and consider the following problem:

$$
\begin{aligned}
& \mathbf{u}_{t}-\Delta \mathbf{u}+c \mathbf{u}_{\xi}-\mathbf{f}(\mathbf{u})=\mathbf{0}, \quad \mathbf{x}^{\prime} \in \mathbb{R}^{n-1}, \quad \xi \in \mathbb{R}, t>0, \\
& \mathbf{u}\left(\mathbf{x}^{\prime}, \xi, 0\right)=\mathbf{u}_{0}\left(\mathbf{x}^{\prime}, \xi\right), \quad \mathbf{x}^{\prime} \in \mathbb{R}^{n-1}, \xi \in \mathbb{R} .
\end{aligned}
$$

The main results of this paper are as follows.

Theorem 1.1. Assume that (H1)-(H2) hold. Let $\mathbf{u}_{0} \in\left[\mathbf{E}^{-}, \mathbf{E}^{+}\right]$be such that

$$
\lim _{R \rightarrow \infty} \sup _{\left|\mathbf{x}^{\prime}\right|+|\xi| \geq R}\left|\mathbf{u}_{0}\left(\mathbf{x}^{\prime}, \xi\right)-\Phi(\xi)\right|=0 .
$$

Then the solution $\mathbf{u}\left(\mathbf{x}^{\prime}, \xi, t\right)$ of (1.3)-(1.4) satisfies

$$
\lim _{t \rightarrow \infty} \sup _{\left(\mathbf{x}^{\prime}, \xi\right) \in \mathbb{R}^{n}}\left|\mathbf{u}\left(\mathbf{x}^{\prime}, \xi, t\right)-\Phi(\xi)\right|=0 .
$$


Theorem 1.2. Assume that (H1)-(H2) hold. Let

$$
\mathbf{u}_{0}\left(\mathbf{x}^{\prime}, \xi\right)=\Phi\left(\xi+v_{0}\left(\mathbf{x}^{\prime}\right)\right)
$$

for some smooth function $v_{0} \in L^{1}\left(\mathbb{R}^{n-1}\right) \cap L^{\infty}\left(\mathbb{R}^{n-1}\right)$. Then one has

$$
\sup _{\left(\mathbf{x}^{\prime}, \xi\right) \in \mathbb{R}^{n}}\left|\mathbf{u}\left(\mathbf{x}^{\prime}, \xi, t\right)-\Phi(\xi)\right| \leq C t^{-\frac{n-1}{2}}, \quad t>0,
$$

where $C>0$ is a constant depending on $\mathbf{f},\left\|v_{0}\right\|_{L^{1}\left(\mathbb{R}^{n-1}\right)}$ and $\left\|v_{0}\right\|_{L^{\infty}\left(\mathbb{R}^{n-1}\right)}$.

Proposition 1.3. Assume that the assumptions in Theorem 1.2 hold. Assume further that either $v_{0} \geq 0$, $v_{0} \not \equiv 0$ or $v_{0} \leq 0, v_{0} \not \equiv 0$. Then there exist constants $\widehat{C}>0$ and $\widetilde{C}>0$ such that

$$
\widehat{C}(1+t)^{-\frac{n-1}{2}} \leq \sup _{\left(\mathbf{x}^{\prime}, \xi\right) \in \mathbb{R}^{n}}\left|\mathbf{u}\left(\mathbf{x}^{\prime}, \xi, t\right)-\Phi(\xi)\right| \leq \widetilde{C} t^{-\frac{n-1}{2}}, \quad t>0 .
$$

Theorem 1.4. Assume that $l \geq\left[\frac{n-1}{2}\right]+1$ and

$$
\Phi\left(\xi-\sigma_{1}^{0}\left(\mathbf{x}^{\prime}\right)\right)-q_{1}^{0}\left(\mathbf{x}^{\prime}\right) \mathbf{p}(\xi) \leq \mathbf{u}_{0}\left(\mathbf{x}^{\prime}, \xi\right) \leq \Phi\left(\xi+\sigma_{2}^{0}\left(\mathbf{x}^{\prime}\right)\right)+q_{2}^{0}\left(\mathbf{x}^{\prime}\right) \mathbf{p}(\xi),
$$

where $\mathbf{p}(\xi)$ is defined in (2.3). Assume that there exists a constant $\delta>0$ such that

$$
E_{0}=\left\|\sigma_{i}^{0}\right\|_{L^{1}}+\left\|\sigma_{i}^{0}\right\|_{H^{l+1}}+\left\|q_{i}^{0}\right\|_{L^{1}}+\left\|q_{i}^{0}\right\|_{H^{l}} \leq \delta \quad \text { for } i=1,2 .
$$

Then there exists a positive constant $D$ satisfying

$$
\begin{aligned}
& \sup _{\left(\mathbf{x}^{\prime}, \xi\right) \in \mathbb{R}^{n}}\left|\mathbf{u}\left(\mathbf{x}^{\prime}, \xi, t\right)-\Phi(\xi)\right| \leq D(1+t)^{-\frac{1}{2}}, \quad n=2,3, t \geq 0, \\
& \sup _{\left(\mathbf{x}^{\prime}, \xi\right) \in \mathbb{R}^{n}}\left|\mathbf{u}\left(\mathbf{x}^{\prime}, \xi, t\right)-\Phi(\xi)\right| \leq D(1+t)^{-\frac{n+1}{4}}, \quad n \geq 4, t \geq 0 .
\end{aligned}
$$

Theorem 1.5. Let $n=2$. Assume that $(\mathrm{H} 1)-(\mathrm{H} 2)$ hold. Let $\mathbf{u}\left(x^{\prime}, \xi, 0\right)=\Phi\left(\xi+v_{0}^{*}\left(x^{\prime}\right)\right)$ for some $v_{0}^{*}\left(x^{\prime}\right)$ defined on $\mathbb{R}$ with $\left\|v_{0}^{*}\right\|_{L^{\infty}(\mathbb{R})}=\delta$. Then for $t_{\omega}=\frac{\omega(\omega !)^{2}}{4}$, there holds

$$
\lim _{\omega \rightarrow \infty} \sup _{\left|x^{\prime}\right| \leq \omega !-1, \xi \in \mathbb{R}}\left|\mathbf{u}\left(x^{\prime}, \xi, t_{\omega}\right)-\Phi\left(\xi+(-1)^{\omega} \delta\right)\right|=0 .
$$

Remark 1.6. In light of Theorems 1.2 and 1.5, the boundedness of the perturbations in $L^{1}$ may play a substantial role in considering the multidimensional stability. Moreover, the results of Theorem 1.4 is new for reaction-diffusion systems. Actually, compared with the results obtained by $[6,13]$, we extend the results in [13] to reaction-diffusion systems and get a convergence rate, and the rate we obtain in Theorem 1.4 is better than $t^{-\frac{n-1}{4}}$ in [6].

The rest of this paper is organized as follows. In Section 2, we state some preliminaries. In Section 3, we prove the multidimensional stability for spatially decaying initial perturbations, i.e., we prove Theorems 1.1 and 1.2 and Proposition 1.3. Furthermore, we show that planar traveling fronts are not always asymptotically stable in multiple dimensions under general bounded initial perturbations in Section 4, namely, we prove Theorem 1.5. The proof of Theorem 1.4 is left in Section 5 .

\section{Preliminaries}

By virtue of Theorems 3.2 and 3.6 and Lemma 3.7 in [4] and Theorem 4.1 in [3], we have the following lemma. 
Lemma 2.1. Assume that (H1)-(H2) hold. Let $\Phi$ be the traveling front defined in (1.2). Then there exist $\lambda>0, \mu<0$ and $A^{-}, A^{+} \in \mathbb{R}_{+}^{m}$ such that

$$
\begin{aligned}
& \Phi(\xi)-E^{-}=A^{-} e^{\lambda \xi}+o\left(e^{\lambda \xi}\right), \quad \xi \rightarrow-\infty, \\
& \Phi^{\prime}(\xi)=A^{-} \lambda e^{\lambda \xi}+o\left(e^{\lambda \xi}\right), \quad \xi \rightarrow-\infty, \\
& \Phi(\xi)-E^{+}=A^{+} e^{\mu \xi}+o\left(e^{\mu \xi}\right), \quad \xi \rightarrow+\infty, \\
& \Phi^{\prime}(\xi)=A^{+} \mu e^{\mu \xi}+o\left(e^{\mu \xi}\right), \quad \xi \rightarrow+\infty .
\end{aligned}
$$

According to Lemma 2.1, we get the following lemma.

Lemma 2.2. Let $\Phi(\xi)$ be the traveling front of (1.2). Then there exists a constant $k>0$ which depends only on $\mathbf{f}$ such that

$$
-k \Phi^{\prime} \leq \Phi^{\prime \prime} \leq k \Phi^{\prime} \quad \text { for } \xi \in \mathbb{R}
$$

Lemma 2.3. Let $v^{ \pm}\left(\mathbf{x}^{\prime}, t\right)$ be solutions of the following problem:

$$
\begin{aligned}
& \frac{\partial}{\partial t} v^{ \pm}\left(\mathbf{x}^{\prime}, t\right)=\Delta_{\mathbf{x}^{\prime}} v^{ \pm} \pm k\left|\nabla_{\mathbf{x}^{\prime}} v^{ \pm}\right|^{2}, \quad \mathbf{x}^{\prime} \in \mathbb{R}^{n-1}, t>0 \\
& v^{ \pm}\left(\mathbf{x}^{\prime}, 0\right)=v_{0}^{ \pm}\left(\mathbf{x}^{\prime}\right), \quad \mathbf{x}^{\prime} \in \mathbb{R}^{n-1},
\end{aligned}
$$

where $k$ is the constant defined as in Lemma 2.2. Let $\mathbf{u}\left(\mathbf{x}^{\prime}, \xi, t\right)$ be the solution of (1.3)-(1.4) with

$$
\Phi\left(\xi+v_{0}^{-}\left(\mathbf{x}^{\prime}\right)\right) \leq \mathbf{u}_{0}\left(\mathbf{x}^{\prime}, \xi\right) \leq \Phi\left(\xi+v_{0}^{+}\left(\mathbf{x}^{\prime}\right)\right), \quad\left(\mathbf{x}^{\prime}, \xi\right) \in \mathbb{R}^{n} .
$$

Then one has

$$
\Phi\left(\xi+v^{-}\left(\mathbf{x}^{\prime}, t\right)\right) \leq \mathbf{u}\left(\mathbf{x}^{\prime}, \xi, t\right) \leq \Phi\left(\xi+v^{+}\left(\mathbf{x}^{\prime}, t\right)\right), \quad\left(\mathbf{x}^{\prime}, \xi\right) \in \mathbb{R}^{n}, t \geq 0
$$

Proof. Define

$$
\mathcal{L}_{i}[\mathbf{u}]:=\frac{\partial u_{i}}{\partial t}-\Delta u_{i}+c \partial_{\xi} u_{i}-\tilde{f}_{i}(\mathbf{u})=0 .
$$

We only show that $\Phi\left(\xi+v^{-}\left(\mathbf{x}^{\prime}, t\right)\right)$ is a subsolution of (1.3), since the supersolution can be proved similarly. It suffices to show that $\mathcal{L}_{i}[\Phi]\left(\xi+v^{-}\left(\mathbf{x}^{\prime}, t\right)\right) \leq 0$. In fact, by Lemma 2.2, we have

$$
\begin{aligned}
\mathcal{L}_{i}[\Phi]\left(\xi+v^{-}\left(\mathbf{x}^{\prime}, t\right)\right) & :=\frac{\partial \phi_{i}}{\partial t}\left(\xi+v^{-}\left(\mathbf{x}^{\prime}, t\right)\right)-\Delta \phi_{i}\left(\xi+v^{-}\left(\mathbf{x}^{\prime}, t\right)\right)+c \partial_{\xi} \phi_{i}-f_{i}\left(\Phi\left(\xi+v^{-}\left(\mathbf{x}^{\prime}, t\right)\right)\right) \\
& =\phi_{i}^{\prime} \frac{\partial v^{-}}{\partial t}-\phi_{i}^{\prime \prime}-\phi_{i}^{\prime \prime}\left|\nabla_{\mathbf{x}^{\prime}} v^{-}\right|-\phi_{i}^{\prime} \Delta_{\mathbf{x}^{\prime}} v^{-}+c \phi_{i}^{\prime}-f_{i}\left(\Phi\left(\xi+v^{-}\left(\mathbf{x}^{\prime}, t\right)\right)\right) \\
& \leq \phi_{i}^{\prime}\left(\frac{\partial v^{-}}{\partial t}-\Delta_{\mathbf{x}^{\prime}} v^{-}+k\left|\nabla_{\mathbf{x}^{\prime}} v^{-}\right|^{2}\right)=0 .
\end{aligned}
$$

This completes the proof.

Lemma 2.4 ([13, Lemmas 2.4 and 2.5]). Let $k>0$ be any constant and $v^{ \pm}\left(\mathbf{x}^{\prime}, t\right)$ be solutions of the following Cauchy problems:

$$
\begin{aligned}
& v_{t}^{ \pm}=\Delta v^{ \pm} \pm k\left|\nabla v^{ \pm}\right|^{2}, \quad \mathbf{x}^{\prime} \in \mathbb{R}^{n-1}, \quad t>0, \\
& v^{ \pm}\left(\mathbf{x}^{\prime}, 0\right)=v_{0}\left(\mathbf{x}^{\prime}\right), \quad \mathbf{x}^{\prime} \in \mathbb{R}^{n-1} .
\end{aligned}
$$

If $v_{0}\left(\mathbf{x}^{\prime}\right)$ is bounded and continuous on $\mathbb{R}^{n-1}$ and satisfies $\lim _{\left|\mathbf{x}^{\prime}\right| \rightarrow \infty}\left|v_{0}\left(\mathbf{x}^{\prime}\right)\right|=0$, then there hold

$$
\lim _{t \rightarrow \infty} \sup _{\mathbf{x}^{\prime} \in \mathbb{R}^{n-1}}\left|v^{ \pm}\left(\mathbf{x}^{\prime}, t\right)\right|=0 .
$$


Moreover, if we further assume that $v_{0} \in L^{1}\left(\mathbb{R}^{n-1}\right)$, then we have

$$
\sup _{\mathbf{x}^{\prime} \in \mathbb{R}^{n-1}}\left|v^{ \pm}\left(\mathbf{x}^{\prime}, t\right)\right| \leq \frac{1}{k}\left\|\exp \left(k v_{0}\right)-1\right\|_{L^{1}\left(\mathbb{R}^{n-1}\right)} \cdot t^{-\frac{n-1}{2}}, \quad t>0 .
$$

Lemma 2.5 ([13, Lemma 2.8]). Assume that $\mathbf{u}_{0}\left(\mathbf{x}^{\prime}, \xi\right)$ satisfies

$$
\lim _{R \rightarrow \infty} \sup _{\left|\mathbf{x}^{\prime}\right|+|\xi| \geq R}\left|\mathbf{u}_{0}\left(\mathbf{x}^{\prime}, \xi\right)-\Phi(\xi)\right|=0 .
$$

Then there holds

$$
\lim _{R \rightarrow \infty} \sup _{\left|\mathbf{x}^{\prime}\right|+|\xi| \geq R}\left|\mathbf{u}\left(\mathbf{x}^{\prime}, \xi, T\right)-\Phi(\xi)\right|=0
$$

for any fixed $T>0$, where $\mathbf{u}\left(\mathbf{x}^{\prime}, \xi, t\right)$ is the solution of (1.3)-(1.4).

Lemma 2.6 ([13, Lemmas 3.1 and 3.2]). Let $n=2$. Let $k>0$ be defined as in Lemma 2.2 and $v^{ \pm}(x, t)$ be solutions of the following problem:

$$
\begin{aligned}
& v_{t}^{ \pm}=v_{x x}^{ \pm} \pm k v^{ \pm}, \quad x \in \mathbb{R}, t>0, \\
& v^{ \pm}(x, 0)=v_{0}^{ \pm}(x), \quad x \in \mathbb{R},
\end{aligned}
$$

respectively. Suppose that $v_{0}^{ \pm}(x)$ are all bounded functions on $\mathbb{R}$ and satisfy

$$
v_{0}^{+}(x) \leq \delta, \quad x \in \mathbb{R}, \quad \text { and } \quad v_{0}^{+}(x) \leq-\delta, \quad|x| \in[\omega !+1,(\omega+1) !-1]
$$

and

$$
v_{0}^{-}(x) \geq-\delta, \quad x \in \mathbb{R}, \quad \text { and } \quad v_{0}^{-}(x) \geq \delta, \quad|x| \in[\omega !+1,(\omega+1) !-1]
$$

for some constant $\delta>0$ and some integer $q \geq 2$, respectively. Then there hold

$$
\sup _{|x| \leq \omega !-1} v^{+}(x, T) \leq-\delta+C \int_{|\zeta| \in[0,2 / \sqrt{\omega}] \cup[\sqrt{\omega}, \infty]} e^{-\zeta^{2}} d \zeta
$$

and

$$
\sup _{|x| \leq \omega !-1} v^{-}(x, T) \geq \delta-C \int_{|\zeta| \in[0,2 / \sqrt{\omega}] \cup[\sqrt{\omega}, \infty]} e^{-\zeta^{2}} d \zeta,
$$

respectively, where $T=\omega(\omega !)^{2} / 4, C>0$ is a constant that only depends on $\delta$ and $k$.

By virtue of the assumption (H1) and Perron-Frobenius theorem there exist irreducible constant matrices $B^{ \pm}=\left(\mu_{i j}^{ \pm}\right)$such that $\frac{\partial f_{i}}{\partial u_{j}}\left(\mathbf{E}^{ \pm}\right)<\mu_{i j}^{ \pm}$for all $i, j=1, \ldots, m$, and that the principal eigenvalues of $B^{ \pm}$are negative. Furthermore, we can choose positive vectors $\mathbf{p}^{ \pm}=\left(p_{1}^{ \pm}, \ldots, p_{m}^{ \pm}\right)$such that $\mathbf{p}^{ \pm}$are the positive eigenvectors corresponding to the principal eigenvalues of $B^{ \pm}$. Define

$$
\zeta(s):=\frac{1}{2}\left(1+\tanh \left(\frac{s}{2}\right)\right) .
$$

Let the positive vector function $\mathbf{p}(\xi):=\left(p_{1}(\xi), \ldots, p_{m}(\xi)\right)$ be defined by

$$
p_{i}(\xi)=\zeta(\xi) p_{i}^{+}+(1-\zeta(\xi)) p_{i}^{-}, \quad i=1, \ldots, m .
$$

It is easy to check that $\mathbf{p}(\xi)$ satisfies

$$
\begin{aligned}
& p_{i}(\cdot) \in\left[\min \left\{p_{i}^{-}, p_{i}^{+}\right\}, \max \left\{p_{i}^{-}, p_{i}^{+}\right\}\right] \quad \text { on } \mathbb{R} \quad \text { and } \quad \min _{1 \leq i \leq m} \inf _{\xi \in \mathbb{R}} p_{i}(\xi)>0, \\
& p_{i}(\xi) \rightarrow p_{i}^{ \pm} \quad \text { as } \xi \rightarrow \pm \infty \quad \text { and } \quad p_{i}^{\prime}(\xi) \rightarrow 0 \quad \text { as } \xi \rightarrow \pm \infty \text { for } i=1, \ldots, m
\end{aligned}
$$


Lemma 2.7. Let $k>0$ be given in Lemma 2.2. Then there exist positive constants $\rho \geq 1$ and $\beta$ such that, for any $\delta \in\left(0, \delta_{0}\right)$, the functions $\mathbf{u}^{ \pm}\left(\mathbf{x}^{\prime}, \xi, t\right)$ defined by

$$
\mathbf{u}^{ \pm}\left(\mathbf{x}^{\prime}, \xi, t\right):=\Phi\left(\xi+v^{ \pm}\left(\mathbf{x}^{\prime}, t\right) \pm \rho \delta\left(1-e^{-\beta t}\right)\right) \pm \delta \mathbf{p}\left(\xi+v^{ \pm}\left(\mathbf{x}^{\prime}, t\right) \pm \rho \delta\left(1-e^{-\beta t}\right)\right) e^{-\beta t}
$$

are a supersolution and a subsolution of (1.3), respectively, where $v^{ \pm}\left(\mathbf{x}^{\prime}, t\right)$ are respective solutions of the following equations:

$$
\begin{aligned}
& v_{t}^{ \pm}=\Delta_{\mathbf{x}^{\prime}} v^{ \pm} \pm k\left|\nabla_{\mathbf{x}^{\prime}} v^{ \pm}\right|^{2}, \quad \mathbf{x}^{\prime} \in \mathbb{R}^{n-1}, t>0 . \\
& v^{ \pm}\left(\mathbf{x}^{\prime}, 0\right)=v_{0}^{ \pm}, \quad \mathbf{x}^{\prime} \in \mathbb{R}^{n-1}
\end{aligned}
$$

with $v_{0}^{ \pm}$are continuous and bounded functions.

Proof. It suffices to prove that $\mathbf{u}^{+}\left(\mathbf{x}^{\prime}, \xi, t\right)$ is a supersolution of (1.3), since the subsolution can be proved in the same way. For $\mathbf{v} \in \mathbb{R}^{m}$ and $r_{1}>0$, set $B_{r_{1}}(\mathbf{v})=\left\{\mathbf{u} \in \mathbb{R}^{m}:\|\mathbf{u}-\mathbf{v}\|<r_{1}\right\}$. By the definitions of $\mu_{i j}^{ \pm}$, there exist a sufficiently small positive constant $\epsilon$ and a positive constant $r$ such that

$$
\begin{aligned}
& \frac{\partial f_{i}}{\partial u_{j}}(\mathbf{u}) \leq \mu_{i j}^{ \pm} \quad \text { for } \mathbf{u} \in B_{4 \epsilon}\left(\mathbf{E}^{ \pm}\right) \cap\left[\mathbf{E}^{-}, \mathbf{E}^{+}\right] \text {and } i, j=1, \ldots, m, \\
& \sum_{j=1}^{m} \mu_{i j}^{ \pm} \gamma_{j} \leq-r \gamma_{i} \quad \text { for } \gamma=\left(\gamma_{1}, \ldots, \gamma_{m}\right) \in B_{4 \epsilon}\left(\mathbf{p}^{ \pm}\right) \cap \mathbb{R}_{+}^{m} .
\end{aligned}
$$

By the parabolic estimates, there exists $M_{1}>0$ such that $\left|\nabla_{\mathbf{x}^{\prime}} v^{ \pm}\right|^{2} \leq M_{1}$. Owing to $\Phi(\xi) \rightarrow \mathbf{E}^{ \pm}$as $\xi \rightarrow \pm \infty$, there exist a sufficiently small positive constant $\delta_{1} \in(0,1 / 2)$ and a sufficiently large constant $M$ such that

$$
\begin{aligned}
& r-(k+1) \delta_{1} M_{1}-2 \delta_{1}>0, \\
& \left|p_{i}^{\prime}(\xi)\right|,\left|p_{i}^{\prime \prime}(\xi)\right| \leq \delta_{1} p_{i}(\xi) \quad \text { for }|\xi| \geq M \text { and } i=1, \ldots, m, \\
& \left\|\mathbf{p}(\xi)-p^{+}\right\| \leq \epsilon \quad \text { for } \xi \geq M, \quad\left\|\mathbf{p}(\xi)-p^{-}\right\| \leq \epsilon \quad \text { for } \xi \leq-M, \\
& \left\|\Phi(\xi)-\mathbf{E}^{+}\right\| \leq \epsilon \quad \text { for } \delta \in\left(0, \delta_{1}\right] \text { and } \xi \geq M \\
& \left\|\Phi(\xi)-\mathbf{E}^{-}\right\| \leq \epsilon \quad \text { for } \delta \in\left(0, \delta_{1}\right] \text { and } \xi \leq-M
\end{aligned}
$$

Set

$$
\begin{aligned}
& \beta \in\left(0, \frac{r-(k+1) \delta_{1} M_{1}-2 \delta_{1}}{1+\delta_{1}}\right), \\
& \delta_{0}:=\min \left\{\delta_{1}, \frac{1}{\rho}, \frac{C_{3}}{2 C_{1}}, \frac{\epsilon}{C_{1}}, \frac{\delta_{1}}{(m-1) C_{1} L^{+}}, \frac{\delta_{1}}{(m-1) C_{1} L^{-}}\right\}, \\
& \rho \geq \max \left\{\frac{2 C_{1}\left(C_{1} C_{2}+C_{1} \beta+(c+1) C_{1}+(k+1) C_{1} M_{1}\right)}{\beta C_{3}}, 1\right\},
\end{aligned}
$$

where $C_{i}, i=1,2,3$ are given by

$$
\begin{aligned}
C_{1} & :=\max \left\{\sup _{\xi \in \mathbb{R}}\|\mathbf{p}(\xi)\|, \sup _{\xi \in \mathbb{R}}\left\|\mathbf{p}^{\prime}(\xi)\right\|, \sup _{\xi \in \mathbb{R}}\left\|\mathbf{p}^{\prime \prime}(\xi)\right\|\right\}, \\
C_{2} & :=\max _{1 \leq i \leq m}\left\{\sup _{\mathbf{u} \in\left[\widehat{\mathbf{E}}^{-}, \widehat{\mathbf{E}}^{+}\right]} \sum_{j=1}^{n}\left|\frac{\partial f_{i}}{\partial u_{j}}(\mathbf{u})\right|\right\}, \\
C_{3} & :=\min _{1 \leq i \leq m}\left\{\inf _{|\xi| \leq M} \phi_{i}^{\prime}(\xi)\right\}, \\
L^{+} & =\max _{i, j=1, \ldots, m} L_{i j}^{+}, \quad L^{-}=\max _{i, j=1, \ldots, m} L_{i j}^{-} .
\end{aligned}
$$


For simplicity, we write $\xi+v^{ \pm}\left(\mathbf{x}^{\prime}, t\right) \pm \rho \delta\left(1-e^{-\beta t}\right)$ by $\eta$. Substituting $\mathbf{u}^{+}$into (2.2), we have

$$
\begin{aligned}
\mathcal{L}_{i}\left[\mathbf{u}^{+}\right]= & \frac{\partial v^{+}}{\partial t} \phi_{i}^{\prime}+\rho \delta \beta e^{-\beta t} \phi_{i}^{\prime}-\delta \beta p_{i} e^{-\beta t}+\frac{\partial v^{+}}{\partial t} \delta e^{-\beta t} p_{i}^{\prime}+\delta e^{-\beta t} \rho \delta \beta e^{-\beta t} p_{i}^{\prime} \\
& -\phi_{i}^{\prime \prime}-\phi_{i}^{\prime \prime}\left|\nabla_{\mathbf{x}^{\prime}} v^{+}\right|^{2}-\phi_{i}^{\prime} \Delta_{\mathbf{x}^{\prime}} v^{+}-\delta e^{-\beta t} p_{i}^{\prime \prime}-\delta e^{-\beta t} p_{i}^{\prime \prime}\left|\nabla_{\mathbf{x}^{\prime}} v^{+}\right|^{2}-\delta e^{-\beta t} p_{i}^{\prime} \Delta_{\mathbf{x}^{\prime}} v^{+} \\
& +c \phi_{i}^{\prime}+c \delta e^{-\beta t} p_{i}^{\prime}-f_{i}\left(\mathbf{u}^{+}\right)-\sum_{1 \leq j \leq m, j \neq i} L_{i j}^{-}\left\{u_{i}^{+}-E_{i}^{-}\right\}^{-}\left(u_{j}^{+}-E_{j}^{-}\right) \\
& -\sum_{1 \leq j \leq m, j \neq i} L_{i j}^{+}\left\{E_{i}^{+}-u_{i}^{+}\right\}^{-}\left(u_{j}^{+}-E_{j}^{+}\right) \\
= & \left(k \phi_{i}^{\prime}-\phi_{i}^{\prime \prime}\right)\left|\nabla_{\mathbf{x}^{\prime}} v^{+}\right|^{2}+\rho \delta \beta e^{-\beta t} \phi_{i}^{\prime}+f_{i}(\Phi)-f_{i}\left(\mathbf{u}^{+}\right) \\
& +\delta e^{-\beta t}\left(-\beta p_{i}+c p_{i}^{\prime}+\left(k p_{i}^{\prime}-p_{i}^{\prime \prime}\right)\left|\nabla_{\mathbf{x}^{\prime}} v^{+}\right|^{2}+\rho \delta \beta e^{-\beta t} p_{i}^{\prime}-p_{i}^{\prime \prime}\right) \\
& -\sum_{1 \leq j \leq m, j \neq i} L_{i j}^{-}\left\{u_{i}^{+}-E_{i}^{-}\right\}^{-}\left(u_{j}^{+}-E_{j}^{-}\right)-\sum_{1 \leq j \leq m, j \neq i} L_{i j}^{+}\left\{E_{i}^{+}-u_{i}^{+}\right\}^{-}\left(u_{j}^{+}-E_{j}^{+}\right) \\
\geq & \rho \delta \beta e^{-\beta t} \phi_{i}^{\prime}+f_{i}(\Phi)-f_{i}\left(\mathbf{u}^{+}\right) \\
& -\delta e^{-\beta t}\left(\beta p_{i}-c p_{i}^{\prime}-\left(k p_{i}^{\prime}-p_{i}^{\prime \prime}\right)\left|\nabla_{\mathbf{x}^{\prime}} v^{+}\right|^{2}-\rho \delta \beta e^{-\beta t} p_{i}^{\prime}+p_{i}^{\prime \prime}\right) \\
& -\sum_{1 \leq j \leq m, j \neq i} L_{i j}^{-}\left\{u_{i}^{+}-E_{i}^{-}\right\}^{-}\left(u_{j}^{+}-E_{j}^{-}\right)-\sum_{1 \leq j \leq m, j \neq i} L_{i j}^{+}\left\{E_{i}^{+}-u_{i}^{+}\right\}-\left(u_{j}^{+}-E_{j}^{+}\right) .
\end{aligned}
$$

To show $\mathcal{L}_{i}\left[\mathbf{u}^{+}\right] \geq 0$ for $i=1, \ldots, m$, we divide $\mathbb{R}$ into three disjoint cases: (i) $|\eta| \leq M$, (ii) $\eta>M$, (iii) $\eta<-M$.

We first consider case (i). Apparently, $\left\{u_{i}^{+}-E_{i}^{-}\right\}^{-}=0$. In view of (2.6), we have $E_{i}^{+}-u_{i}^{+} \geq 0$ due to $\delta \leq \frac{\epsilon}{C_{1}}$ from (2.7), whence $\left\{E_{i}^{+}-u_{i}^{+}\right\}^{-}=0$. It follows from the mean value theorem that there exists $\theta_{i}=\theta_{i}(\mathbf{x}, t) \in(0,1)$ such that

$$
f_{i}(\Phi)-f_{i}\left(\mathbf{u}^{+}\right)=-\sum_{j=1}^{m} \frac{\partial f_{i}}{\partial u_{j}}\left(\Phi+\theta_{i} \delta \mathbf{p} e^{-\beta t}\right) \delta p_{j} e^{-\beta t} \geq-C_{1} C_{2} \delta e^{-\beta t}
$$

By (2.6), (2.7) and (2.9), we obtain

$$
\begin{aligned}
\mathcal{L}_{i}\left[\mathbf{u}^{+}\right] \geq & -C_{1} C_{2} \delta e^{-\beta t}+C_{3} \rho \delta \beta e^{-\beta t} \\
& -\delta e^{-\beta t}\left(C_{1} \beta+\rho \delta \beta e^{-\beta t} C_{1}+(c+1) C_{1}+(k+1) C_{1} M_{1}\right) \\
\geq & \delta e^{-\beta t}\left[\rho \beta\left(C_{3}-\delta C_{1}\right)-\left(C_{1} C_{2}+C_{1} \beta+(c+1) C_{1}+(k+1) C_{1} M_{1}\right)\right] \\
\geq & 0 .
\end{aligned}
$$

Now we turn to case (ii). It is clear that $\left\{u_{i}^{+}-E_{i}^{-}\right\}^{-}=0$. Moreover, the definition of $\mathbf{u}^{+}$implies $\left\{E_{i}^{+}-\right.$ $\left.u_{i}^{+}\right\}^{-} \leq \delta p_{i} e^{-\beta t}$. By the mean value theorem and (2.4)-(2.6), there exists $\theta_{i}=\theta_{i}(\mathbf{x}, t) \in(0,1)$ such that

$$
\begin{aligned}
f_{i}(\Phi)-f_{i}\left(\mathbf{u}^{+}\right) & =-\sum_{j=1}^{m} \frac{\partial f_{i}}{\partial u_{j}}\left(\Phi+\theta_{i} \delta \mathbf{p} e^{-\beta t}\right) \delta p_{j} e^{-\beta t} \\
& \geq-\delta e^{-\beta t} \sum_{j=1}^{m} \mu_{i j}^{+} p_{j} \geq r \delta p_{i} e^{-\beta t} .
\end{aligned}
$$

It then follows from $(2.7)-(2.9)$ that

$$
\begin{aligned}
\mathcal{L}_{i}\left[\mathbf{u}^{+}\right] & \geq r \delta e^{-\beta t} p_{i}(\eta)-\delta e^{-\beta t} p_{i}(\eta)\left(\beta+(k+1) \delta_{1} M_{1}+\rho \delta \beta \delta_{1}+\delta_{1}\right)-\sum_{1 \leq j \leq m, j \neq i} L_{i j}^{+}\left\{E_{i}^{+}-u_{i}^{+}\right\}^{-}\left(u_{j}^{+}-E_{j}^{+}\right) \\
& \geq r \delta e^{-\beta t} p_{i}(\eta)-\delta e^{-\beta t} p_{i}(\eta)\left(\beta+(k+1) \delta_{1} M_{1}+\rho \delta \beta \delta_{1}+\delta_{1}+\delta(m-1) C_{1} L^{+}\right) \\
& \geq \delta e^{-\beta t} p_{i}(\eta)\left(r-\beta-(k+1) \delta_{1} M_{1}-\beta \delta_{1}-2 \delta_{1}\right) \geq 0 .
\end{aligned}
$$

Similarly, we can prove $\mathcal{L}_{i}\left[\mathbf{u}^{+}\right] \geq 0$ for case (iii). This completes the proof. 
The following lemma comes from Lemma 3.2 in [6], see also [5,7,2,20].

Lemma 2.8. Let $\mathbf{x}^{\prime} \in \mathbb{R}^{n-1}$ and $l \geq\left[\frac{n-1}{2}\right]+1$. Then the semigroup $S(t)$ generated by the linear operator $\mathcal{N}=\Delta_{\mathbf{x}^{\prime}}$ enjoys the following decay estimates:

(i) $\|S(t) u\|_{H^{l}\left(\mathbb{R}^{n-1}\right)} \leq A_{1}\|u\|_{H^{l}\left(\mathbb{R}^{n-1}\right)}$,

(ii) $\|S(t) u\|_{H^{l}\left(\mathbb{R}^{n-1}\right)} \leq A_{1}(1+t)^{-\frac{n-1}{4}}\|u\|_{L^{1}\left(\mathbb{R}^{n-1}\right)}+A_{1} e^{-\nu t}\|u\|_{H^{l}\left(\mathbb{R}^{n-1}\right)}$,

(iii) $\left\|\nabla_{\mathbf{x}^{\prime}}(S(t) u)\right\|_{H^{l}\left(\mathbb{R}^{n-1}\right)} \leq A_{1} t^{-\frac{1}{2}}\|u\|_{H^{l}\left(\mathbb{R}^{n-1}\right)}$,

(iv) $\left\|\nabla_{\mathbf{x}^{\prime}}(S(t) u)\right\|_{H^{l}\left(\mathbb{R}^{n-1}\right)} \leq A_{1}(1+t)^{-\frac{n+1}{4}}\|u\|_{L^{1}\left(\mathbb{R}^{n-1}\right)}+A_{1} t^{-\frac{1}{2}} e^{-\nu t}\|u\|_{H^{l}\left(\mathbb{R}^{n-1}\right)}$,

where $A_{1}$ and $\nu$ are positive constants and $\nu$ is independent of $u$.

\section{Asymptotic stability under spatially decaying perturbations}

In light of the supersolutions and subsolutions constructed in Section 2, we prove Theorems 1.1 and 1.2 and Proposition 1.3 in this section.

Proof of Theorem 1.1. We only show the upper estimate, since the lower estimate can be proved similarly. Take constants $k>0$ as in Lemma 2.2 and $\rho \geq 1$ as in (2.7). Let constants $\delta_{0}>\varepsilon>0$ and $\hat{\varepsilon}=\varepsilon /\left(2\left\|\Phi^{\prime}\right\|_{L^{\infty}(\mathbb{R})}+C_{1}\right)$ with $C_{1}$ defined as in (2.8). By the comparison principle, we have

$$
\mathbf{E}^{-} \leq \mathbf{u}\left(\mathbf{x}^{\prime}, \xi, t\right) \leq \mathbf{E}^{+} .
$$

It then follows from the assumption (1.5) and Lemma 2.5 that there exists a constant $R>0$ such that

$$
\sup _{\left|\mathbf{x}^{\prime}\right|+|\xi| \geq R}\left|\mathbf{u}\left(\mathbf{x}^{\prime}, \xi, T_{1}\right)-\Phi(\xi)\right| \leq \frac{\hat{\varepsilon}}{\rho} \min _{1 \leq i \leq n}\left\{\inf _{s \in \mathbb{R}} p(s)\right\}, \quad\left(\mathbf{x}^{\prime}, \xi\right) \in \mathbb{R}^{n}
$$

for $T_{1}>0$. Thus we can take a function $v_{0}\left(\mathbf{x}^{\prime}\right) \geq 0$ with $\lim _{\left|\mathbf{x}^{\prime}\right| \rightarrow \infty} v_{0}\left(\mathbf{x}^{\prime}\right)=0$ such that

$$
\mathbf{u}\left(\mathbf{x}^{\prime}, \xi, T_{1}\right) \leq \Phi\left(\xi+v_{0}\left(\mathbf{x}^{\prime}\right)\right)+\frac{\hat{\varepsilon}}{\rho} \min _{1 \leq i \leq n}\left\{\inf _{s \in \mathbb{R}} p(s)\right\} \mathbf{1}, \quad\left(\mathbf{x}^{\prime}, \xi\right) \in \mathbb{R}^{n} .
$$

Then the comparison principle and the supersolution constructed in Lemma 2.7 imply

$$
\mathbf{u}\left(\mathbf{x}^{\prime}, \xi, t\right) \leq \Phi\left(\xi+v\left(\mathbf{x}^{\prime}, t\right)+\hat{\varepsilon}\left(1-e^{-\beta t}\right)\right)+\frac{\hat{\varepsilon}}{\rho} \mathbf{p}\left(\xi+v\left(\mathbf{x}^{\prime}, t\right)+\hat{\varepsilon}\left(1-e^{-\beta t}\right)\right) e^{-\beta t},
$$

where $v\left(\mathbf{x}^{\prime}, t\right)$ is the solution of the following problem:

$$
\begin{aligned}
& v_{t}=\Delta_{\mathbf{x}^{\prime}} v+k\left|\nabla_{\mathbf{x}^{\prime}} v\right|^{2}, \quad \mathbf{x}^{\prime} \in \mathbb{R}^{n-1}, t>0, \\
& v\left(\mathbf{x}^{\prime}, 0\right)=v_{0}\left(\mathbf{x}^{\prime}\right), \quad \mathbf{x}^{\prime} \in \mathbb{R}^{n-1} .
\end{aligned}
$$

In view of Lemma 2.4, we have that there exists a constant $T_{2}>0$ such that $v\left(\mathbf{x}^{\prime}, T_{2}\right) \leq \hat{\varepsilon}$ for $\mathbf{x}^{\prime} \in \mathbb{R}^{n-1}$. It then follows that

$$
\mathbf{u}\left(\mathbf{x}^{\prime}, \xi, t\right) \leq \Phi(\xi+2 \hat{\varepsilon})+C_{1} \hat{\varepsilon} \mathbf{1} \leq \Phi(\xi)+\left(2\left\|\Phi^{\prime}\right\|_{L^{\infty}(\mathbb{R})}+C_{1}\right) \hat{\varepsilon} \mathbf{1}=\Phi(\xi)+\varepsilon \mathbf{1}
$$

for $t \geq T_{1}+T_{2}$ and $\left(\mathbf{x}^{\prime}, \xi\right) \in \mathbb{R}^{n}$, where $C_{1}$ is given in (2.8). By using a similar argument, one can obtain

$$
\mathbf{u}\left(\mathbf{x}^{\prime}, \xi, t\right) \geq \Phi(\xi-2 \hat{\varepsilon})-C_{1} \hat{\varepsilon} \mathbf{1} \geq \Phi(\xi)-\left(2\left\|\Phi^{\prime}\right\|_{L^{\infty}(\mathbb{R})}+C_{1}\right) \hat{\varepsilon} \mathbf{1}=\Phi(\xi)+\varepsilon \mathbf{1}
$$

for $t \geq T_{1}+T_{2}$ and $\left(\mathbf{x}^{\prime}, \xi\right) \in \mathbb{R}^{n}$. Combining the above two inequalities, we get the conclusion of Theorem 1.1. The proof is complete. 
Proof of Theorem 1.2. In light of Lemma 2.3, one has

$$
\begin{aligned}
\Phi(\xi)-\left\|\Phi^{\prime}\right\|_{L^{\infty}(\mathbb{R})} \cdot \sup _{\mathbf{x}^{\prime} \in \mathbb{R}^{n-1}}\left|v^{-}\left(\mathbf{x}^{\prime}, t\right)\right| \mathbf{1} & \leq \Phi\left(\xi+v^{-}\left(\mathbf{x}^{\prime}, t\right)\right) \\
& \leq \mathbf{u}\left(\mathbf{x}^{\prime}, \xi, t\right) \\
& \leq \Phi\left(\xi+v^{+}\left(\mathbf{x}^{\prime}, t\right)\right) \\
& \leq \Phi(\xi)+\left\|\Phi^{\prime}\right\|_{L^{\infty}(\mathbb{R})} \cdot \sup _{\mathbf{x}^{\prime} \in \mathbb{R}^{n-1}}\left|v^{+}\left(\mathbf{x}^{\prime}, t\right)\right| \mathbf{1} .
\end{aligned}
$$

Hence, the statement of Theorem 1.2 is an immediate consequence of Lemma 2.4. This completes the proof.

Proof of Proposition 1.3. We only study the case $v_{0} \geq 0, v_{0} \not \equiv 0$, since the case $v_{0} \leq 0, v_{0} \not \equiv 0$ can be treated similarly. By Theorem 1.2, it remains to show that

$$
\sup _{\left(\mathbf{x}^{\prime}, \xi\right) \in \mathbb{R}^{n}}\left|\mathbf{u}\left(\mathbf{x}^{\prime}, \xi, t\right)-\Phi(\xi)\right| \geq \widehat{C}_{1}(1+t)^{-\frac{n-1}{2}} .
$$

Following from Cole-Hopf transformation, we obtain that

$$
v\left(\mathbf{x}^{\prime}, t\right)=-\frac{1}{k} \ln \left(\int_{\mathbb{R}^{n-1}} \Gamma\left(\mathbf{x}^{\prime}-\mathbf{y}, t\right) \exp \left(-k v_{0}(\mathbf{y})\right) d \mathbf{y}\right)
$$

is the solution of the following problem

$$
\begin{aligned}
& v_{t}=\Delta_{\mathbf{x}^{\prime}} v-k\left|\nabla_{\mathbf{x}^{\prime}} v\right|^{2}, \quad \mathbf{x}^{\prime} \in \mathbb{R}^{n-1}, t>0, \\
& v\left(\mathbf{x}^{\prime}, 0\right)=v_{0}\left(\mathbf{x}^{\prime}\right), \quad \mathbf{x}^{\prime} \in \mathbb{R}^{n-1},
\end{aligned}
$$

where $\Gamma(a, b)$ is defined by

$$
\Gamma(a, b)=\frac{1}{(4 \pi b)^{\frac{n-1}{2}}} e^{-\frac{|a|^{2}}{4 b}} .
$$

The assumption $v_{0} \geq 0, v_{0} \not \equiv 0$ implies that there exist a constant $\delta>0$ and a nonempty open set $D \subset \mathbb{R}^{n-1}$ such that $v_{0} \geq \delta$ for $\mathbf{x}^{\prime} \in D$. It then follows that

$$
\begin{aligned}
v\left(\mathbf{x}^{\prime}, t\right) & \geq-\frac{1}{k} \ln \left(1-\int_{D} \Gamma\left(\mathbf{x}^{\prime}-\mathbf{y}, t\right)(1-\exp (-k \delta)) d \mathbf{y}\right) \\
& \geq-\frac{1}{k} \ln \left(1-|D|(1-\exp (-k \delta)) \cdot \min _{\mathbf{y} \in D} \Gamma\left(\mathbf{x}^{\prime}-\mathbf{y}, t\right)\right) \\
& \geq \frac{|D|}{k}(1-\exp (-k \delta)) \cdot \min _{\mathbf{y} \in D} \Gamma\left(\mathbf{x}^{\prime}-\mathbf{y}, t\right),
\end{aligned}
$$

which implies $v(\mathbf{0}, t) \geq C^{\prime}(1+t)^{-\frac{n-1}{2}}$. By the first inequality of (2.1), we have

$$
\begin{aligned}
\mathbf{u}(\mathbf{0}, t) & \geq \Phi(v(\mathbf{0}, t)) \geq \Phi(0)+\min _{\xi \in\left[0,\|v\|_{L^{\infty}\left(\mathbb{R}^{n-1}\right)}\right]}\left|\Phi^{\prime}(\xi)\right| \cdot v(\mathbf{0}, t) \mathbf{1} \\
& \geq \Phi(0)+C^{\prime} \mathbf{1}(1+t)^{-\frac{n-1}{2}}
\end{aligned}
$$

for $t \geq 0$. Thus we obtain the left-hand inequality of (1.6). This completes the proof.

\section{Permanent oscillating solutions}

In this section, we show Theorem 1.5, which indicates that planar traveling fronts are not always stable in multiple dimensions. For convenience, we write $x$ instead of $x^{\prime}$ in the sequel. 
Proof of Theorem 1.5. We define two sequences of smooth functions $\left\{v_{0, i}^{ \pm}(x)\right\}_{i=1,2, \ldots}$ satisfying

$$
\left|v_{0, i}^{ \pm}(x)\right| \leq \delta, \quad x \in \mathbb{R}, \quad v_{0, i}^{+}(x)=\left\{\begin{array}{l}
-\delta, \quad|x| \in I_{2 i}, \quad \text { and } \quad v_{0, i}^{-}(x)=\left\{\begin{array}{l}
\delta, \quad|x| \in I_{2 i+1}, \\
\delta, \quad|x| \in \tilde{I}_{2 i},
\end{array}-|x| \in \tilde{I}_{2 i+1},\right.
\end{array}\right.
$$

where

$$
I_{\omega}=[\omega !+1,(\omega+1) !-1], \quad \tilde{I}_{\omega}=[0, \omega !] \cup[(\omega+1) !, \infty] .
$$

Let $v_{0}^{*}(x) \in C^{\infty}(\mathbb{R})$ be such that

$$
v_{0, i}^{-}(x) \leq v_{0}^{*}(x) \leq v_{0, i}^{+}(x) \text { for all } i \geq 1 .
$$

Set $\mathbf{u}^{*}(x, \xi, t)$ be the solution of $(1.3)-(1.4)$ with $\mathbf{u}^{*}(x, \xi, 0)=\Phi\left(\xi+v_{0}^{*}(x)\right)$ and $v_{i}^{ \pm}(x, t)$ be the solution of the following problem:

$$
\begin{aligned}
& v_{i, t}^{ \pm}=v_{i, x x}^{ \pm} \pm k\left(v_{i, x}^{ \pm}\right)^{2}, \quad x \in \mathbb{R}, t>0, \\
& v_{i}^{ \pm}(x, 0)=v_{0, i}^{ \pm}(x), \quad x \in \mathbb{R} .
\end{aligned}
$$

It then follows from the comparison principle that

$$
\Phi(\xi-\delta) \leq \Phi\left(\xi+v_{0, i}^{-}(x)\right) \leq \Phi\left(\xi+v_{0}^{*}(x)\right) \leq \Phi\left(\xi+v_{0, i}^{+}(x)\right) \leq \Phi(\xi+\delta) .
$$

Moreover, Lemma 2.3 yields that

$$
\Phi(\xi-\delta) \leq \mathbf{u}^{*}(x, \xi, t) \leq \Phi\left(\xi+v_{i}^{+}(x, t)\right) .
$$

In view of Lemma 2.6, we get

$$
\begin{aligned}
\Phi(\xi-\delta) & \leq \sup _{|x| \leq(2 i) !-1} \mathbf{u}^{*}\left(x, \xi, t_{2 i}\right) \leq \sup _{|x| \leq(2 i) !-1} \Phi\left(\xi+v_{i}^{+}\left(x, t_{2 i}\right)\right) \\
& \leq \Phi(\xi-\delta)+\left\|\Phi^{\prime}\right\|_{L^{\infty}(\mathbb{R})} \cdot C \mathbf{1} \int_{|\vartheta| \in[0,2 / \sqrt{2 i}] \cup[\sqrt{2 i}, \infty]} e^{-\vartheta^{2}} d \vartheta,
\end{aligned}
$$

where $t_{2 i}=(2 i)((2 i) !)^{2} / 4$. This implies that

$$
\lim _{i \rightarrow \infty} \sup _{|x| \leq(2 i) !-1, \xi \in \mathbb{R}}\left|\mathbf{u}^{*}\left(x, \xi, t_{2 i}\right)-\Phi(\xi-\delta)\right|=0 .
$$

On the other hand, by Lemma 2.3 and the inequality $v_{0}^{*}(x) \geq v_{0, i}^{-}(x)$ for $i=1,2, \ldots$, we have

$$
\begin{aligned}
\Phi(\xi+\delta) & \geq \sup _{|x| \leq(2 i+1) !-1} \mathbf{u}^{*}\left(x, \xi, t_{2 i+1}\right) \geq \sup _{|x| \leq(2 i+1) !-1} \Phi\left(\xi+v_{i}^{-}\left(x, t_{2 i+1}\right)\right) \\
& \geq \Phi(\xi+\delta)-\left\|\Phi^{\prime}\right\|_{L^{\infty}(\mathbb{R})} \cdot C \mathbf{1} \int_{|\vartheta| \in[0,2 / \sqrt{2 i+1}] \cup[\sqrt{2 i+1}, \infty]} e^{-\vartheta^{2}} d \vartheta
\end{aligned}
$$

where $t_{2 i+1}=(2 i+1)((2 i+1) !)^{2} / 4$. Thus we have

$$
\lim _{i \rightarrow \infty} \sup _{|x| \leq(2 i+1) !-1, \xi \in \mathbb{R}}\left|\mathbf{u}^{*}\left(x, \xi, t_{2 i+1}\right)-\Phi(\xi+\delta)\right|=0 .
$$

The conclusion of Theorem 1.5 follows from (4.1)-(4.2). The proof is complete.

\section{Proof of Theorem 1.4}

In this section, we obtain explicit estimates for $\sigma\left(\mathbf{x}^{\prime}, t\right)$ and $q\left(\mathbf{x}^{\prime}, t\right)$ by appealing to the theory of semigroup. In other words, we prove Theorem 1.4. We use the same notations as in the previous sections. 
Lemma 5.1. Let $\sigma\left(\mathbf{x}^{\prime}, t\right)$ and $q\left(\mathbf{x}^{\prime}, t\right)$ be solutions of the following problems:

$$
\begin{aligned}
& \frac{\partial \sigma}{\partial t}-\Delta_{\mathbf{x}^{\prime}} \sigma+a\left|\nabla_{\mathbf{x}^{\prime}} \sigma\right|^{2}-\alpha q=0, \quad \mathbf{x}^{\prime} \in \mathbb{R}^{n-1}, t>0 \\
& \sigma\left(\mathbf{x}^{\prime}, 0\right)=\sigma_{1}^{0}\left(\mathbf{x}^{\prime}\right), \quad \mathbf{x}^{\prime} \in \mathbb{R}^{n-1}
\end{aligned}
$$

and

$$
\begin{aligned}
& \frac{\partial q}{\partial t}-\Delta_{\mathbf{x}^{\prime}} q+\frac{r}{2} q=0, \quad \mathbf{x}^{\prime} \in \mathbb{R}^{n-1}, t>0 \\
& q\left(\mathbf{x}^{\prime}, 0\right)=q_{1}^{0}\left(\mathbf{x}^{\prime}\right), \quad \mathbf{x}^{\prime} \in \mathbb{R}^{n-1}
\end{aligned}
$$

respectively, where

$$
a=\max \left\{c-2 \kappa_{0}, c-2 \kappa_{1}, c-\kappa_{2}\right\} \quad \text { and } \quad \alpha=\frac{C_{1}\left(\kappa_{2}+\frac{r}{2}+1+c\right)}{C_{3}}
$$

with $C_{1}, C_{3}$ defined as in (2.8) and $\kappa_{0}, \kappa_{1}, \kappa_{2}$ specified in the sequel. Then the functions defined by

$$
\overline{\mathbf{u}}\left(\mathbf{x}^{\prime}, \xi, t\right):=\Phi\left(\xi+\sigma\left(\mathbf{x}^{\prime}, t\right)\right)+q\left(\mathbf{x}^{\prime}, t\right) \mathbf{p}(\xi)
$$

and

$$
\underline{\mathbf{u}}\left(\mathbf{x}^{\prime}, \xi, t\right):=\Phi\left(\xi-\sigma\left(\mathbf{x}^{\prime}, t\right)\right)-q\left(\mathbf{x}^{\prime}, t\right) \mathbf{p}(\xi)
$$

is a pair of super-subsolutions of (1.3).

Proof. It suffices to show $\underline{\mathbf{u}}\left(\mathbf{x}^{\prime}, \xi, t\right)$ is a subsolution, since the supersolution can be proved analogously. Noting that $\left\{E_{i}^{+}-\underline{u}_{i}\right\}^{-}=0$, substituting $\underline{\mathbf{u}}$ into (2.2), we have

$$
\begin{aligned}
\mathcal{L}_{i}[\underline{\mathbf{u}}]= & \frac{\partial \underline{u}_{i}}{\partial t}-\Delta \underline{u}_{i}+c \partial_{\xi} \underline{u}_{i}-f_{i}(\underline{\mathbf{u}})-\sum_{1 \leq j \leq m, j \neq i} L_{i j}^{-}\left\{\underline{u}_{i}-E_{i}^{-}\right\}^{-}\left(\underline{u}_{j}-E_{j}^{-}\right) \\
= & -\phi_{i}^{\prime} \frac{\partial \sigma}{\partial t}-\phi_{i}^{\prime \prime}-\phi_{i}^{\prime \prime}\left|\nabla_{\mathbf{x}^{\prime}} \sigma\right|^{2}+\phi_{i}^{\prime} \Delta_{\mathbf{x}^{\prime}} \sigma+c \phi_{i}^{\prime}-f_{i}(\underline{\mathbf{u}})-\left(\frac{\partial q_{i}}{\partial t}-\Delta_{\mathbf{x}^{\prime}} q_{i}\right) p_{i}(\xi)-q\left(p_{i}^{\prime \prime}+c p_{i}^{\prime}\right) \\
& -\sum_{1 \leq j \leq m, j \neq i} L_{i j}^{-}\left\{\underline{u}_{i}-E_{i}^{-}\right\}^{-}\left(\underline{u}_{j}-E_{j}^{-}\right) \\
= & -\phi_{i}^{\prime}\left(\frac{\partial \sigma}{\partial t}-\Delta_{\mathbf{x}^{\prime}} \sigma+c\left|\nabla_{\mathbf{x}^{\prime}} \sigma\right|^{2}\right)+f_{i}(\Phi)\left|\nabla_{\mathbf{x}^{\prime}} \sigma\right|^{2}-\left(f_{i}(\underline{\mathbf{u}})-f_{i}(\Phi)\right) \\
& -\left(\frac{\partial q}{\partial t}-\Delta_{\mathbf{x}^{\prime}} q\right) p_{i}(\xi)-q\left(p_{i}^{\prime \prime}+c p_{i}^{\prime}\right)-\sum_{1 \leq j \leq m, j \neq i} L_{i j}^{-}\left\{\underline{u}_{i}-E_{i}^{-}\right\}^{-}\left(\underline{u}_{j}-E_{j}^{-}\right) .
\end{aligned}
$$

By the definition of $p_{i}(\xi)$ in (2.3), there exists a constant $M$ sufficiently large such that (2.4)-(2.6) hold and $\max _{i=1, \ldots, m} \max _{\xi \in \mathbb{R}}\left\{\left|\phi_{i}^{\prime}\right|\right\}\|\sigma\|_{L^{\infty}} \leq \epsilon$ when $\xi<-M$ or $\xi>M$. Moreover, when $\xi<-M$ or $\xi>M$, we choose $q \in\left(0, \frac{\epsilon}{C_{1}}\right)$, then one has

$$
\mathbf{E}^{-}-3 \epsilon \mathbf{1} \leq \Phi(\xi)-\sigma \max _{i=1, \ldots, m} \max _{\xi \in \mathbb{R}}\left\{\left|\phi_{i}^{\prime}\right|\right\}-\theta_{i} q \mathbf{p} \leq \Phi(\xi-\sigma)-\theta_{i} q \mathbf{p} \leq \Phi(\xi)-q \mathbf{p} \leq \mathbf{E}^{-}+2 \epsilon \mathbf{1} .
$$

Thanks to the mean value theorem, there exists $\theta_{i}\left(\mathbf{x}^{\prime}, t\right) \in[0,1]$ such that

$$
f_{i}(\underline{\mathbf{u}})-f_{i}(\Phi)=-\sum_{j=1}^{m} \frac{\partial f_{i}}{\partial u_{j}}\left(\Phi-\theta_{i} q \mathbf{p}\right) q p_{j} \geq-q \sum_{j=1}^{m} \mu_{i j}^{+} p_{j} \geq r q p_{i} .
$$

We consider three cases: (i) $\xi<-M$, (ii) $\xi>M$, (iii) $|\xi|<M$. 
(i) $\xi<-M$. By virtue of Lemma 2.1 and (2.8), there exists a positive constant $\kappa_{0}$ satisfying

$$
\lim _{\xi \rightarrow-\infty} \frac{f_{i}(\Phi)}{\phi_{i}^{\prime}}=\sum_{j=1}^{m} \frac{\partial f_{i}}{\partial u_{j}}\left(\mathbf{E}^{-}\right) \frac{\phi_{j}-E_{j}^{-}}{\phi_{i}^{\prime}} \leq \kappa_{0} .
$$

On the other hand, one deduces

$$
\sum_{1 \leq j \leq m, j \neq i} L_{i j}^{-}\left\{\underline{u}_{i}-E_{i}^{-}\right\}^{-}\left(\underline{u}_{j}-E_{j}^{-}\right) \geq-\sum_{1 \leq j \leq m, j \neq i} L_{i j}^{-} q^{2} p_{i} p_{j} \geq-(m-1) L^{-} q^{2} p_{i} C_{1} \geq-\frac{1}{4} r q p_{i}
$$

by taking $q \leq \frac{r}{4(m-1) L^{-} C_{1}}$. Since $p_{i}^{\prime} \rightarrow 0$ and $p_{i}^{\prime \prime} \rightarrow 0$ as $\xi \rightarrow-\infty$, then there hold

$$
-q\left(p_{i}^{\prime \prime}+c p_{i}^{\prime}\right) \leq \frac{1}{4} r q p_{i}
$$

Hence, we have

$$
\mathcal{L}_{i}[\underline{\mathbf{u}}] \leq-\phi_{i}^{\prime}\left(\frac{\partial \sigma}{\partial t}-\Delta_{\mathbf{x}^{\prime}} \sigma+\left(c-2 \kappa_{0}\right)\left|\nabla_{\mathbf{x}^{\prime}} \sigma\right|^{2}\right)-\left(\frac{\partial q}{\partial t}-\Delta_{\mathbf{x}^{\prime}} q+\frac{r}{2} q\right) p_{i} \leq 0 .
$$

(ii) $\xi>M$. Owing to Lemma 2.1, we have

$$
\lim _{\xi \rightarrow \infty} \frac{f_{i}(\Phi)}{\phi_{i}^{\prime}}=-\sum_{j=1}^{m} \frac{\partial f_{i}}{\partial u_{j}}\left(\mathbf{E}^{+}\right) \frac{E_{j}^{+}-\phi_{j}}{\phi_{i}^{\prime}} \leq \kappa_{1}
$$

for some positive constant $\kappa_{1}$. Thus by a similar argument to case (i) one infers

$$
\mathcal{L}_{i}[\underline{\mathbf{u}}] \leq-\phi_{i}^{\prime}\left(\frac{\partial \sigma}{\partial t}-\Delta_{\mathbf{x}^{\prime}} \sigma+\left(c-2 \kappa_{1}\right)\left|\nabla_{\mathbf{x}^{\prime}} \sigma\right|^{2}\right)-\left(\frac{\partial q}{\partial t}-\Delta_{\mathbf{x}^{\prime}} q+\frac{r}{2} q\right) p_{i} \leq 0 .
$$

(iii) $|\xi| \leq M$. In view of (2.8), we have $\phi_{i}^{\prime}(\xi) \geq C_{3}$ for $|\xi| \leq M$. By the hypothesis (H2), there exists $\kappa_{2}>0$ such that $f_{i}(\Phi) \leq \kappa_{2} \phi_{i}^{\prime}$ and

$$
f_{i}(\Phi)-f_{i}(\Phi-q \mathbf{p}(\xi))=\sum_{j=1}^{m} \frac{\partial}{\partial u_{j}} f_{i}\left(\Phi-\theta_{i} q \mathbf{p}(\xi)\right) q p_{j} \leq \kappa_{2} q p_{i} .
$$

It then follows from (2.6) and (2.8) that

$$
\begin{aligned}
\mathcal{L}_{i}[\underline{\mathbf{u}}] & \leq-\phi_{i}^{\prime}\left(\frac{\partial \sigma}{\partial t}-\Delta_{\mathbf{x}^{\prime}} \sigma+\left(c-\kappa_{2}\right)\left|\nabla_{\mathbf{x}^{\prime}} \sigma\right|^{2}\right)+\left(\kappa_{2}+r\right) q p_{i}-q\left(p_{i}^{\prime \prime}+c p_{i}^{\prime}\right)-\left(\frac{\partial q}{\partial t}-\Delta_{\mathbf{x}^{\prime}} q+r q\right) p_{i} \\
& \leq-\phi_{i}^{\prime}\left(\frac{\partial \sigma}{\partial t}-\Delta_{\mathbf{x}^{\prime}} \sigma+\left(c-\kappa_{2}\right)\left|\nabla_{\mathbf{x}^{\prime}} \sigma\right|^{2}\right)+C_{1}\left(\kappa_{2}+\frac{r}{2}+1+c\right) q-\left(\frac{\partial q}{\partial t}-\Delta_{\mathbf{x}^{\prime}} q+\frac{r}{2} q\right) p_{i} \\
& =0 .
\end{aligned}
$$

Combining (5.3)-(5.5), we get the conclusion. The proof is complete.

Lemma 5.2. There exists a unique solution $q\left(\mathbf{x}^{\prime}, t\right)$ of (5.2) such that

$$
\left\|q\left(\mathbf{x}^{\prime}, t\right)\right\|_{H^{l}\left(\mathbb{R}^{n-1}\right)} \leq e^{-\frac{r}{2} t}\left\|q_{0}\right\|_{H^{l}\left(\mathbb{R}^{n-1}\right)}
$$

and

$$
\left\|q\left(\mathbf{x}^{\prime}, t\right)\right\|_{L^{1}\left(\mathbb{R}^{n-1}\right)} \leq e^{-\frac{r}{2} t}\left\|q_{0}\right\|_{L^{1}\left(\mathbb{R}^{n-1}\right)} .
$$

Proof. Rewrite equation (5.2) as

$$
\partial_{t}\left(e^{\frac{r}{2} t} q\right)=\Delta_{\mathbf{x}^{\prime}}\left(e^{\frac{r}{2} t} q\right), \quad \mathbf{x}^{\prime} \in \mathbb{R}^{n-1}, t>0 .
$$


Then there exists a unique solution of the form

$$
q\left(\mathbf{x}^{\prime}, t\right)=e^{-\frac{r}{2} t} S(t) q_{0},
$$

where $S(t)$ is the semigroup generated by the operator $\Delta_{\mathbf{x}^{\prime}}$. Moreover, it follows from Lemma 2.8 that for $l \geq\left[\frac{n-1}{2}\right]+1$,

$$
\|q(t)\|_{H^{l}\left(\mathbb{R}^{n-1}\right)} \leq e^{-\frac{r}{2} t}\left\|q_{0}\right\|_{H^{l}\left(\mathbb{R}^{n-1}\right)} .
$$

Since $\|S(t) h\|_{L^{1}} \leq\|h\|_{L^{1}}$ for any function $h$, then we have

$$
\|q(t)\|_{L^{1}\left(\mathbb{R}^{n-1}\right)} \leq e^{-\frac{r}{2} t}\left\|q_{0}\right\|_{L^{1}\left(\mathbb{R}^{n-1}\right)} .
$$

The proof is complete.

Lemma 5.3. If $\sigma\left(\mathbf{x}^{\prime}, t\right)$ is a solution of $(5.1)$, then $w\left(\mathbf{x}^{\prime}, t\right)=\nabla_{\mathbf{x}^{\prime}} \sigma\left(\mathbf{x}^{\prime}, t\right)$ satisfies

$$
\sup _{0 \leq t \leq 1}\left\|w\left(\mathbf{x}^{\prime}, t\right)\right\|_{H^{l}} \leq 2 A_{1}\left\|\sigma_{1}^{0}\left(\mathbf{x}^{\prime}\right)\right\|_{H^{l+1}}+4 \alpha A_{1}\|q(t)\|_{H^{l}} .
$$

Proof. Taking the divergence to the first equation of problem (5.1), we get

$$
\begin{aligned}
& \partial_{t} w-\Delta_{\mathbf{x}^{\prime}} w-\nabla_{\mathbf{x}^{\prime}} \cdot\left(a|w|^{2}+\alpha q\right)=0, \quad \mathbf{x}^{\prime} \in \mathbb{R}^{n-1}, t>0, \\
& w\left(\mathbf{x}^{\prime}, 0\right)=\nabla_{\mathbf{x}^{\prime}} \sigma_{0}\left(\mathbf{x}^{\prime}\right), \quad \mathbf{x}^{\prime} \in \mathbb{R}^{n-1} .
\end{aligned}
$$

We a priori assume that

$$
M(t) \equiv \sup _{0 \leq \tau \leq t}(1+\tau)^{\frac{n+1}{4}}\|w(\tau)\|_{H^{l}\left(\mathbb{R}^{n-1}\right)} \leq M_{0}, \quad t \geq 0
$$

for some constant $M_{0} \leq \frac{1}{4 a A_{1} A_{2}}$. Notice that the Sobolev imbedding yields

$$
\left\|h_{1} h_{2}\right\|_{H^{l}\left(\mathbb{R}^{n-1}\right)} \leq A_{2}\left\|h_{1}\right\|_{H^{l}\left(\mathbb{R}^{n-1}\right)}\left\|h_{2}\right\|_{H^{l}\left(\mathbb{R}^{n-1}\right)}
$$

for any functions $h_{1}, h_{2}$, where $A_{2}$ is a positive constant depending only on $l$ and $n$.

Since $\nabla_{\mathbf{x}^{\prime}}(S(t) h)=S(t) \nabla_{\mathbf{x}^{\prime}} h$ for any function $h$, then the solution of problem (5.9) can be written as

$$
w\left(\mathbf{x}^{\prime}, t\right)=S(t)\left(\nabla_{\mathbf{x}^{\prime}} \sigma_{0}\left(\mathbf{x}^{\prime}\right)\right)-\int_{0}^{t} \nabla_{\mathbf{x}^{\prime}}\left(S(t-\tau)\left(a|w|^{2}(\tau)+\alpha q(\tau)\right)\right) d \tau .
$$

It then follows from Lemma 2.8 and (5.11) that for $l \geq\left[\frac{n-1}{2}\right]+1$,

$$
\left\|w\left(\mathbf{x}^{\prime}, t\right)\right\|_{H^{l}\left(\mathbb{R}^{n-1}\right)} \leq A_{1}\left\|\nabla_{\mathbf{x}^{\prime}} \sigma_{0}\left(\mathbf{x}^{\prime}\right)\right\|_{H^{l}\left(\mathbb{R}^{n-1}\right)}+A_{1} \int_{0}^{t}(t-\tau)^{-\frac{1}{2}}\left(a A_{2}\|w(\tau)\|_{H^{l}\left(\mathbb{R}^{n-1}\right)}^{2}+\alpha\|q(\tau)\|_{H^{l}\left(\mathbb{R}^{n-1}\right)}\right) d \tau
$$

This yields that for $l \geq\left[\frac{n-1}{2}\right]+1$,

$$
\begin{aligned}
& \left\|w\left(\mathbf{x}^{\prime}, t\right)\right\|_{H^{l}\left(\mathbb{R}^{n-1}\right)} \\
& \quad \leq A_{1}\left\|\sigma_{0}\left(\mathbf{x}^{\prime}\right)\right\|_{H^{l+1}\left(\mathbb{R}^{n-1}\right)}+2 \alpha A_{1} t^{1 / 2}\|q(t)\|_{H^{l}\left(\mathbb{R}^{n-1}\right)}+2 a A_{1} A_{2} t^{1 / 2}\left(\sup _{0 \leq \tau \leq t}\left\|w\left(\mathbf{x}^{\prime}, \tau\right)\right\|_{H^{l}\left(\mathbb{R}^{n-1}\right)}\right)^{2} \\
& \quad \leq A_{1}\left\|\sigma_{0}\left(\mathbf{x}^{\prime}\right)\right\|_{H^{l+1}\left(\mathbb{R}^{n-1}\right)}+2 \alpha A_{1}\|q(t)\|_{H^{l}\left(\mathbb{R}^{n-1}\right)}+2 a A_{1} A_{2}\left(\sup _{0 \leq t \leq 1}\left\|w\left(\mathbf{x}^{\prime}, t\right)\right\|_{H^{l}\left(\mathbb{R}^{n-1}\right)}\right)^{2} .
\end{aligned}
$$

This together with (5.10) yields

$$
\left(1-2 a A_{1} A_{2} M(t)\right) \sup _{0 \leq t \leq 1}\left\|w\left(\mathbf{x}^{\prime}, t\right)\right\|_{H^{l}} \leq A_{1}\left\|\sigma_{0}\left(\mathbf{x}^{\prime}\right)\right\|_{H^{l+1}}+2 \alpha A_{1}\|q(t)\|_{H^{l}} .
$$


Notice that $M_{0} \leq \frac{1}{4 a A_{1} A_{2}}$. Then we get

$$
\sup _{0 \leq t \leq 1}\left\|w\left(\mathbf{x}^{\prime}, t\right)\right\|_{H^{l}} \leq 2 A_{1}\left\|\sigma_{0}\left(\mathbf{x}^{\prime}\right)\right\|_{H^{l+1}}+4 \alpha A_{1}\|q(t)\|_{H^{l}} .
$$

The proof is complete.

Lemma 5.4. Assume that $w\left(\mathbf{x}^{\prime}, t\right)$ satisfies (5.9). Then one has

$$
(1+t)^{\frac{n+1}{4}}\left\|w\left(\mathbf{x}^{\prime}, t\right)\right\|_{H^{l}} \leq B_{1}\left(\left\|\sigma_{0}\right\|_{L^{1}}+\left\|\sigma_{0}\right\|_{H^{l}}+\left\|q_{0}\right\|_{L^{1}}+\left\|q_{0}\right\|_{H^{l}}\right)+B_{2}(M(t))^{2}
$$

for all $t \geq 1$, where $B_{1}$ and $B_{2}$ are positive constants that will be specified in the sequel.

Proof. Since $\nabla_{\mathbf{x}^{\prime}}(S(t) h)=S(t) \nabla_{\mathbf{x}^{\prime}} h$ for any function $h$, we then write the solution of $(5.9)$ as

$$
w\left(\mathbf{x}^{\prime}, t\right)=\nabla_{\mathbf{x}^{\prime}}\left(S(t) \sigma_{0}\right)-\int_{0}^{t} \nabla_{\mathbf{x}^{\prime}}\left(S(t-\tau)\left(a|w|^{2}(\tau)+\alpha q(\tau)\right)\right) d \tau .
$$

It then follows from Lemma 2.8 and (5.11) that

$$
\begin{aligned}
&\left\|w\left(\mathbf{x}^{\prime}, t\right)\right\|_{H^{l}} \\
& \leq A_{1}\left[(1+t)^{-\frac{n+1}{4}}\left\|\sigma_{0}\right\|_{L^{1}}+t^{-\frac{1}{2}} e^{-\nu t}\left\|\sigma_{0}\right\|_{H^{l}}\right]+A_{1} \int_{0}^{t}(1+t-\tau)^{-\frac{n+1}{4}}\left(a\|w(\tau)\|_{L^{2}}^{2}+\alpha\|q(\tau)\|_{L^{1}}\right) d \tau \\
&+A_{1} \int_{0}^{t}(t-\tau)^{-\frac{1}{2}} e^{-\nu(t-\tau)}\left(a A_{2}\|w(\tau)\|_{H^{l}}^{2}+\alpha\|q(\tau)\|_{H^{l}}\right) d \tau \\
&:=I+I I+I I I .
\end{aligned}
$$

A straightforward computation yields

$$
I \leq A_{3}(1+t)^{-\frac{n+1}{4}}\left(\left\|\sigma_{0}\right\|_{L^{1}}+\left\|\sigma_{0}\right\|_{H^{l}}\right), \quad t \geq 1,
$$

where

$$
A_{3}=A_{1} \max \left\{1, \gamma_{1}\right\} \quad \text { and } \quad \gamma_{1}=e^{\nu}\left(\frac{n+1}{4 \nu e}\right)^{\frac{n+1}{4}} .
$$

By virtue of (5.7) and (5.10), one has

$$
I I \leq a A_{1}(M(t))^{2} \int_{0}^{t}(1+t-\tau)^{-\frac{n+1}{4}}(1+\tau)^{-\frac{n+1}{2}} d \tau+\alpha A_{1}\left\|q_{0}\right\|_{L^{1}} \int_{0}^{t}(1+t-\tau)^{-\frac{n+1}{4}} e^{-\frac{r}{2} \tau} d \tau .
$$

Since

$$
\int_{t / 2}^{t}(1+t-\tau)^{-\frac{n+1}{4}} d \tau \leq\left\{\begin{array}{l}
4\left(1+\frac{t}{2}\right)^{\frac{1}{4}}, \quad n=2, \\
\ln \left(1+\frac{t}{2}\right), \quad n=3, \\
\frac{4}{n-3}, \quad n \geq 4,
\end{array}\right.
$$

then we have

$$
\left(1+\frac{t}{2}\right)^{-\frac{n+1}{4}} \int_{t / 2}^{t}(1+t-\tau)^{-\frac{n+1}{4}} d \tau \leq 4 .
$$

Hence, for $n \geq 2$ and $t \geq 1$, one arrives at

$$
\left(\int_{0}^{t / 2}+\int_{t / 2}^{t}\right)(1+t-\tau)^{-\frac{n+1}{4}}(1+\tau)^{-\frac{n+1}{2}} d \tau \leq \frac{2}{n-1}\left(1+\frac{t}{2}\right)^{-\frac{n+1}{4}}+4\left(1+\frac{t}{2}\right)^{-\frac{n+1}{4}}
$$


and

$$
\begin{aligned}
\left(\int_{0}^{t / 2}+\int_{t / 2}^{t}\right)(1+t-\tau)^{-\frac{n+1}{4}} e^{-\frac{r}{2} \tau} d \tau & \leq \frac{2}{r}\left(1+\frac{t}{2}\right)^{-\frac{n+1}{4}}+4\left(e^{-\frac{r}{2} t}\left(1+\frac{t}{2}\right)^{\frac{n+1}{2}}\right)\left(1+\frac{t}{2}\right)^{-\frac{n+1}{4}} \\
& \leq \frac{2}{r}\left(1+\frac{t}{2}\right)^{-\frac{n+1}{4}}+A_{4}\left(1+\frac{t}{2}\right)^{-\frac{n+1}{4}}
\end{aligned}
$$

where $A_{4}=4 e^{\frac{r}{2}}\left(\frac{n+1}{r e}\right)^{\frac{n+1}{2}}$. It then follows that

$$
(1+t)^{\frac{n+1}{4}} I I \leq A_{5}(M(t))^{2}+A_{6}\left\|q_{0}\right\|_{L^{1}}
$$

for $t \geq 1$, where $A_{5}=a A_{1}\left(4+\frac{2}{n-1}\right) 2^{\frac{n+1}{4}}$ and $A_{6}=\alpha A_{1}\left(\frac{2}{r}+A_{4}\right) 2^{\frac{n+1}{4}}$.

It remains to estimate III. In view of (5.6) and (5.10), we get

$$
I I I \leq a A_{1} A_{2}(M(t))^{2} \int_{0}^{t}(t-\tau)^{-\frac{1}{2}} e^{-\nu(t-\tau)}(1+\tau)^{-\frac{n+1}{2}} d \tau+\alpha A_{1}\left\|q_{0}\right\|_{H^{l}} \int_{0}^{t}(t-\tau)^{-\frac{1}{2}} e^{-\nu(t-\tau)} e^{-\frac{r}{2} \tau} d \tau .
$$

Notice that for $n \geq 2$ and $t \geq 1$, there hold

$$
\left(\int_{0}^{t / 2}+\int_{t / 2}^{t}\right)(t-\tau)^{-\frac{1}{2}} e^{-\nu(t-\tau)}(1+\tau)^{-\frac{n+1}{2}} d \tau \leq \frac{2 \sqrt{2}}{n-1} e^{-\frac{\nu t}{2}}+2\left(1+\frac{t}{2}\right)^{-\frac{n}{2}} \leq A_{7}\left(1+\frac{t}{2}\right)^{-\frac{n+1}{4}}
$$

and

$$
\left(\int_{0}^{t / 2}+\int_{t / 2}^{t}\right)(t-\tau)^{-\frac{1}{2}} e^{-\nu(t-\tau)} e^{-r \tau} d \tau \leq \frac{2 \sqrt{2}}{r} e^{-\frac{\nu}{2} t}+2 \sqrt{\frac{t}{2}} e^{-\frac{r}{2} t} \leq\left(\frac{2 \sqrt{2}}{r} \gamma_{1}+2 A_{8}\right)\left(1+\frac{t}{4}\right)^{-\frac{n+1}{4}},
$$

where $A_{7}=\frac{2 \sqrt{2}}{n-1} \gamma_{1}+2$ and $A_{8}=\left(\frac{n+3}{2 r e}\right)^{\frac{n+3}{4}}$. It then follows that

$$
(1+t)^{\frac{n+1}{4}} I I I \leq A_{9}(M(t))^{2}+A_{10}\left\|q_{0}\right\|_{H^{l}}
$$

for $t \geq 1$, where $A_{9}=2^{\frac{n+1}{4}} a A_{1} A_{2} A_{7}$ and $A_{10}=2^{\frac{n+1}{4}} \alpha A_{1}\left(\frac{2 \sqrt{2} \gamma_{1}}{r}+2 A_{8}\right)$. Consequently, by (5.14)-(5.16), one derives

$$
(1+t)^{\frac{n+1}{4}}\left\|w\left(\mathbf{x}^{\prime}, t\right)\right\|_{H^{l}} \leq B_{1}\left(\left\|\sigma_{0}\right\|_{L^{1}}+\left\|\sigma_{0}\right\|_{H^{l}}+\left\|q_{0}\right\|_{L^{1}}+\left\|q_{0}\right\|_{H^{l}}\right)+B_{2}(M(t))^{2},
$$

where $B_{1}=\max \left\{A_{3}, A_{6}, A_{10}\right\}$ and $B_{2}=A_{5}+A_{9}$. The proof is complete.

Lemma 5.5. Let $M(t)$ be defined as in (5.10). Then one has

$$
M(t) \leq 2 B_{3} E_{0}, \quad t \geq 0 .
$$

Proof. In view of (5.8), we have

$$
\sup _{0 \leq t \leq 1}(1+t)^{\frac{n+1}{4}}\left\|w\left(\mathbf{x}^{\prime}, t\right)\right\|_{H^{l}} \leq 2^{\frac{n+1}{4}}\left(2 A_{1}\left\|\sigma_{0}\right\|_{H^{l+1}}+4 \alpha C_{1}\left\|q_{0}\right\|_{H^{l}}\right) .
$$

It follows from (5.12) that for $t \geq 0$,

$$
M(t) \leq B_{3} E_{0}+B_{2}(M(t))^{2},
$$

where $B_{3}=2^{\frac{n+1}{4}} \max \left\{2 A_{1}, 4 \alpha A_{1}\right\}+B_{1}$. It is evident that $M(t)$ is sufficiently small. Actually, inequality (5.17) holds for all $t \geq 0$ as $1-4 B_{2} B_{3} E_{0} \leq 0$. However, the assumption (1.8) implies that it is impossible by letting $\delta<\frac{1}{8 B_{2} B_{3}}$. Then one obtains

$$
M(t) \leq 2 B_{3} E_{0}, \quad t \geq 0,
$$


or equivalently,

$$
\left\|w\left(\mathbf{x}^{\prime}, t\right)\right\|_{H^{l}} \leq 2 B_{3} E_{0}(1+t)^{-\frac{n+1}{4}}, \quad t \geq 0 .
$$

This completes the proof.

Lemma 5.6. There holds

$$
\|\sigma(t)\|_{H^{l}} \leq\left(B_{9} E_{0}^{2}+B_{10} E_{0}\right)(1+t)^{-\frac{n-1}{4}}, \quad t \geq 0,
$$

where $B_{9}$ and $B_{10}$ will be defined later.

Proof. Write the solution of problem (5.1) as

$$
\sigma(t)=S(t) \sigma_{0}-\int_{0}^{t} S(t-\tau)\left(a|w(\tau)|^{2}+\alpha q(\tau)\right) d \tau .
$$

It follows from (5.11) and Lemma 2.8 that

$$
\begin{aligned}
\|\sigma(t)\|_{H^{l}} \leq & A_{1}\left[(1+t)^{-\frac{n-1}{4}}\left\|\sigma_{0}\right\|_{L^{1}}+e^{-\nu t}\left\|\sigma_{0}\right\|_{H^{l}}\right]+A_{1} \int_{0}^{t}(1+t-\tau)^{-\frac{n-1}{4}}\left(a\|w(\tau)\|_{L^{2}}^{2}+\alpha\|q(\tau)\|_{L^{1}}\right) \\
& +A_{1} \int_{0}^{t} e^{-\nu(t-\tau)}\left(a A_{2}\|w(\tau)\|_{H^{l}}^{2}+\alpha\|q(\tau)\|_{H^{l}}\right) \\
:= & I_{1}+I I_{1}+I I I_{1} .
\end{aligned}
$$

By a similar argument to that of (5.13), we obtain

$$
I_{1} \leq B_{4}(1+t)^{-\frac{n-1}{4}}\left(\left\|\sigma_{0}\right\|_{L^{1}}+\left\|\sigma_{0}\right\|_{H^{l}}\right),
$$

where $B_{4}=A_{1} \max \left\{1, \gamma_{2}\right\}$ and $\gamma_{2}=e^{\nu}\left(\frac{n-1}{4 \nu e}\right)^{\frac{n-1}{4}}$. By virtue of Lemma 5.5, we have

$$
\left(1+\frac{t}{2}\right)^{\frac{n-1}{4}} I I_{1} \leq B_{5}\left(E_{0}\right)^{2}+B_{6}\left\|q_{0}\right\|_{L^{1}},
$$

where $B_{5}=4 a A_{1} B_{3}^{2}\left(4+\frac{2}{n-1}\right)$ and $B_{6}=\alpha A_{1}\left(\frac{2}{r}+A_{4}\right)$. In light of Lemma 5.5 , one gets

$$
\left(1+\frac{t}{2}\right)^{\frac{n-1}{4}} I I I_{1} \leq B_{7}\left(E_{0}\right)^{2}+B_{8}\left\|q_{0}\right\|_{H^{l}},
$$

where $B_{7}=4 a A_{1} A_{2} B_{3}\left(\frac{2 \gamma_{2}}{n-1}+\frac{1}{\beta}\right), B_{8}=\alpha A_{1}\left(\frac{2 \gamma_{2}}{r}+\frac{\gamma_{3}}{\beta}\right)$ and $\gamma_{3}=e^{\frac{r}{2}}\left(\frac{n-1}{2 r e}\right)^{\frac{n-1}{4}}$. We then conclude that for all $t \geq 0$,

$$
\|\sigma(t)\|_{H^{l}} \leq\left(B_{9} E_{0}+B_{10}\right) E_{0}(1+t)^{-\frac{n-1}{4}},
$$

where $B_{9}=2^{\frac{n-1}{4}}\left(B_{5}+B_{7}\right)$ and $B_{10}=\max \left\{B_{4}, 2^{\frac{n-1}{4}} \max \left\{B_{6}, B_{8}\right\}\right\}$. The proof is complete.

Armed with the estimates (5.18) and (5.19), the existence and uniqueness of the solution to the Cauchy problems (5.1) is now a standard application of semigroup theory (see [14]), we omit the details here.

Proof of Theorem 1.4. Let $\sigma_{1}\left(\mathbf{x}^{\prime}, t\right)$ and $q_{1}\left(\mathbf{x}^{\prime}, t\right)$ be solutions of problems (5.1) and (5.2), respectively. It follows from Lemma 5.1 and (1.7) that

$$
\Phi\left(\xi-\sigma_{1}\left(\mathbf{x}^{\prime}, t\right)\right)-q_{1}\left(\mathbf{x}^{\prime}, t\right) \mathbf{p}(\xi) \leq \mathbf{u}\left(\xi, \mathbf{x}^{\prime}, t\right) .
$$

Since $\sigma_{0}\left(\mathbf{x}^{\prime}\right)$ and $q_{0}\left(\mathbf{x}^{\prime}\right)$ are positive, $\sigma_{1}\left(\mathbf{x}^{\prime}, t\right)$ and $q_{1}\left(\mathbf{x}^{\prime}, t\right)$ are always positive. Hence,

$$
\mathbf{u}\left(\xi, \mathbf{x}^{\prime}, t\right)-\Phi(\xi) \geq-\left(\sigma_{1}\left(\mathbf{x}^{\prime}, t\right) \max _{1 \leq i \leq m} \max _{\xi \in \mathbb{R}} \phi_{i}^{\prime}(\xi) \mathbf{1}+q_{1}\left(\mathbf{x}^{\prime}, t\right) \mathbf{p}(\xi)\right) .
$$


Similarly, we can construct the supersolution $\Phi\left(\xi+\sigma_{2}\left(\mathbf{x}^{\prime}, t\right)\right)+q_{2}\left(\mathbf{x}^{\prime}, t\right) \mathbf{p}(\xi)$ such that

$$
\mathbf{u}\left(\mathbf{x}^{\prime}, \xi, t\right)-\Phi(\xi) \leq \sigma_{2}\left(\mathbf{x}^{\prime}, t\right) \max _{1 \leq i \leq m} \max _{\xi \in \mathbb{R}} \phi_{i}^{\prime}(\xi) \mathbf{1}+q_{2}\left(\mathbf{x}^{\prime}, t\right) \mathbf{p}(\xi) .
$$

Consequently, one has

$$
\left|\mathbf{u}\left(\mathbf{x}^{\prime}, \xi, t\right)-\Phi(\xi)\right| \leq \max _{1 \leq i \leq m} \max _{\xi \in \mathbb{R}} \phi_{i}^{\prime}(\xi) \max \left\{\sigma_{1}\left(\mathbf{x}^{\prime}, t\right), \sigma_{2}\left(\mathbf{x}^{\prime}, t\right)\right\}+C_{1} \max \left\{\left|q_{1}\left(\mathbf{x}^{\prime}, t\right)\right|,\left|q_{2}\left(\mathbf{x}^{\prime}, t\right)\right|\right\},
$$

where $C_{1}$ is defined as in (2.8).

In the case $n=2$, it follows from Lemmas 5.2-5.6 and $\|h\|_{L^{\infty}(\mathbb{R})} \leq\|h\|_{L^{2}(\mathbb{R})}^{\frac{1}{2}}\left\|h^{\prime}\right\|_{L^{2}(\mathbb{R})}^{\frac{1}{2}}$ (see [9] for instance) for any function $h$ that there exists some positive constant $D$ such that

$$
\sup _{\left(\mathbf{x}^{\prime}, \xi\right) \in \mathbb{R} \times \mathbb{R}}\left|u\left(\mathbf{x}^{\prime}, \xi, t\right)-\Phi(\xi)\right| \leq D(1+t)^{-\frac{1}{2}} .
$$

In the case $n=3$, the Sobolev embedding implies that $\|h\|_{L^{\infty}\left(\mathbb{R}^{n-1}\right)} \leq D_{0}\|h\|_{W^{l, p}\left(\mathbb{R}^{n-1}\right)}$ when $l p>n-1$ for any function $h$, where $D_{0}$ is a positive constant independent of $h$. It then yields from Lemmas 5.2-5.6 that

$$
\sup _{\left(\mathbf{x}^{\prime}, \xi\right) \in \mathbb{R} \times \mathbb{R}^{2}}\left|u\left(\mathbf{x}^{\prime}, \xi, t\right)-\Phi(\xi)\right| \leq D(1+t)^{-\frac{n-1}{4}}=D(1+t)^{-\frac{1}{2}}
$$

In the case $n \geq 4$, since Lemmas 5.3-5.5 imply that $\sigma \in H^{l+1}\left(\mathbb{R}^{n-1}\right)$, then $\sigma \in W^{l, p}\left(\mathbb{R}^{n-1}\right)$ with $p=\frac{2(n-1)}{n-3}$. By Gagliardo-Nirenberg-Sobolev inequality, that is,

$$
D_{1}\|h\|_{L^{p}\left(\mathbb{R}^{n-1}\right)} \leq\|\nabla h\|_{L^{2}\left(\mathbb{R}^{n-1}\right)}, \quad p=\frac{2(n-1)}{n-3}, n \geq 4,
$$

where $D_{1}$ is a constant depending only on $n$, one infers from Lemmas $5.3-5.5$ that

$$
\|\sigma\|_{W^{l, p}\left(\mathbb{R}^{n-1}\right)} \leq D_{2}\|\nabla \sigma\|_{H^{l}\left(\mathbb{R}^{n-1}\right)} \leq D_{2}(1+t)^{-\frac{n+1}{4}}, \quad p=\frac{2(n-1)}{n-3}
$$

for some constant $D_{2}$ independent of $\sigma$. Hence, in view of Lemmas 5.2-5.6, one has

$$
\sup _{\left(\mathbf{x}^{\prime}, \xi\right) \in \mathbb{R} \times \mathbb{R}^{n-1}}\left|u\left(\mathbf{x}^{\prime}, \xi, t\right)-\Phi(\xi)\right| \leq D(1+t)^{-\frac{n+1}{4}}, \quad n \geq 4 .
$$

Then Theorem 1.4 follows from (5.20)-(5.22). This completes the proof.

\section{Acknowledgments}

We are very grateful to the referee and the editors for their valuable comments and suggestions that helped to improve the original manuscript. The author would like to give his sincere thanks to China Scholarship Council for a one year visit of Aix Marseille University. This paper was supported by NSF of China (11401134).

\section{References}

[1] H. Chen, R. Yuan, Multidimensional stability of disturbed pyramidal traveling fronts in the Allen-Cahn equation, Discrete Contin. Dyn. Syst. Ser. B 20 (2015) 1015-1029.

[2] I.L. Chern, T.P. Liu, Convergence to diffusion waves of solutions for viscous conservation laws, Comm. Math. Phys. 110 (1987) 503-517.

[3] E.A. Coddington, N. Levinson, Theory of Ordinary Differential Equations, McGraw Hill, New York, 1955.

[4] E.C.M. Crooks, On the Vol'pert theory of travelling-wave solutions for parabolic systems, Nonlinear Anal. 26 (1996) 1621-1642.

[5] T. Kapitula, On the nonlinear stability of plane waves for the Ginzburg-Landau equation, Comm. Pure Appl. Math. 47 (1994) 831-841. 
[6] T. Kapitula, Multidimensional stability of planar traveling waves, Trans. Amer. Math. Soc. 349 (1997) $257-269$.

[7] S. Kawashima, Large-time behavior of solutions to hyperbolic-parabolic systems of conservation laws and applications, Proc. Roy. Soc. Edinburgh Sect. A 106 (1987) 169-194.

[8] C.D. Levermore, J. Xin, Multidimensional stability of traveling waves in a bistable reaction-diffusion equation II, Comm. Partial Differential Equations 17 (1992) 1901-1924.

[9] E.H. Lieb, M. Loss, Analysis, in: Graduate Studies in Mathematics, vol. 14, American Mathematical Society, Providence, RI, 2001.

[10] G. Lv, M. Wang, Stability of planar waves in monostable reaction-diffusion equations, Proc. Amer. Math. Soc. 139 (2011) 3611-3621.

[11] G.Y. Lv, M.X. Wang, Stability of planar waves in reaction-diffusion system, Sci. China Math. 54 (2011) $1403-1419$.

[12] H. Matano, M. Nara, Large time behavior of disturbed planar fronts in the Allen-Cahn equation, J. Differential Equations 251 (2011) 3522-3557.

[13] H. Matano, M. Nara, M. Taniguchi, Stability of planar waves in the Allen-Cahn equation, Comm. Partial Differential Equations 34 (2009) 976-1002.

[14] A. Pazy, Semigroups of Linear Operators and Applications to Partial Differential Equations, Springer-Verlag, New York, 1983.

[15] J.M. Roquejoffre, V. Roussier-Michon, Nontrivial large-time behaviour in bistable reaction-diffusion equations, Ann. Mat. Pura Appl. 188 (2009) 207-233.

[16] W.J. Sheng, Multidimensional stability of time-periodic planar traveling fronts in bistable reaction-diffusion equations, Discrete Contin. Dyn. Syst. 37 (2017) 2681-2704.

[17] W.J. Sheng, W.T. Li, Z.C. Wang, Multidimensional stability of V-shaped traveling fronts in the Allen-Cahn equation, Sci. China Math. 56 (2013) 1969-1982.

[18] A.I. Volpert, V.A. Volpert, V.A. Volpert, Travelling Wave Solutions of Parabolic Systems, in: Translations of Mathematical Monographs, vol. 140, American Mathematical Society, Providence, RI, 1994.

[19] Z.C. Wang, Traveling curved fronts in monotone bistable systems, Discrete Contin. Dyn. Syst. 32 (2012) $2339-2374$.

[20] J. Xin, Multidimensional stability of traveling waves in a bistable reaction-diffusion equation I, Comm. Partial Differential Equations 17 (1992) 1889-1899.

[21] H.H. Zeng, Stability of planar travelling waves for bistable reaction-diffusion equations in multiple dimensions, Appl. Anal. 93 (2014) 653-664. 